(2) Open Access Full Text Article

\title{
Comparative efficacy of inhaled medications (ICS/LABA, LAMA, LAMA/LABA and SAMA) for COPD: a systematic review and network meta-
} analysis

This article was published in the following Dove Press journal: International Journal of COPD

\author{
Mohamed Ismail Abdul \\ Aziz ${ }^{1, *}$ \\ Ling Eng Tan ${ }^{1, *}$ \\ David Bin-Chia Wu' \\ Fiona Pearce \\ Gerald Seng Wee Chua ${ }^{2}$ \\ Liang Lin' \\ Ping-Tee Tan' \\ Kwong $\mathrm{Ng}^{\prime}$ \\ 'Agency for Care Effectiveness, \\ Ministry of Health, Singapore; \\ ${ }^{2}$ Division of Medicine, $\mathrm{Ng}$ Teng Fong \\ General Hospital, Singapore \\ *These authors contributed equally \\ to this work
}

\begin{abstract}
Purpose: To assess the comparative efficacy of short-acting muscarinic antagonists (SAMAs), long-acting muscarinic antagonists (LAMAs), LAMA in combination with long-acting betaagonists (LABAs; LAMA/LABAs) and inhaled corticosteroids (ICS) in combination with LABA (ICS/LABAs) for the maintenance treatment of COPD.

Materials and methods: We systematically reviewed 74 randomized controlled trials $(74,832$ participants) published up to 15 November 2017, which compared any of the interventions (SAMA [ipratropium], LAMA [aclidinium, glycopyrronium, tiotropium, umeclidinium], LAMA/LABA [aclidinium/formoterol, indacaterol/glycopyrronium, tiotropium/olodaterol, umeclidinium/vilanterol] and ICS/LABA [fluticasone/vilanterol, budesonide/formoterol, salmeterol/fluticasone]) with each other or with placebo. A random-effects network meta-analysis combining direct and indirect evidence was conducted to examine the change from baseline in trough $\mathrm{FEV}_{1}$, transition dyspnea index, St George's Respiratory Questionnaire and frequency of adverse events at weeks 12 and 24.
\end{abstract}

Results: Inconsistency models were not statistically significant for all outcomes. LAMAs, LAMA/LABAs and ICS/LABAs led to a significantly greater improvement in trough FEV compared with placebo and SAMA monotherapy at weeks 12 and 24. All LAMA/LABAs, except aclidinium/formoterol, were statistically significantly better than LAMA monotherapy and ICS/ LABAs in improving trough $\mathrm{FEV}_{1}$. Among the LAMAs, umeclidinium showed statistically significant improvement in trough $\mathrm{FEV}_{1}$ at week 12 compared to tiotropium and glycopyrronium, but the results were not clinically significant. LAMA/LABAs had the highest probabilities of being ranked the best agents in $\mathrm{FEV}_{1}$ improvement. Similar trends were observed for the transition dyspnea index and St George's Respiratory Questionnaire outcomes. There were no significant differences in the incidences of adverse events among all treatment options.

Conclusion: LAMA/LABA showed the greatest improvement in trough $\mathrm{FEV}_{1}$ at weeks 12 and 24 compared with the other inhaled drug classes, while SAMA showed the least improvement. There were no significant differences among the LAMAs and LAMA/LABAs within their respective classes.

Keywords: anticholinergics, muscarinic antagonists, frequentist meta-analysis, mixed treatment comparison, indirect treatment comparison, chronic obstructive pulmonary disease

\section{Introduction}

COPD is a chronic disorder characterized by fixed airway obstruction with accompanying respiratory symptoms such as persistent and progressive breathlessness, chronic productive cough and limited exercise capacity. It is predominantly caused by 
smoking; however, other factors, particularly occupational exposures, may also contribute to the development of COPD. The impairment of lung function is usually progressive and is not fully reversible. Exacerbations often occur, where there is a rapid and sustained worsening of symptoms beyond normal day-to-day variations. COPD is a global health problem that causes substantial morbidity and mortality. It is the fourth leading cause of death worldwide. ${ }^{1}$

Current disease management guidelines developed by GOLD recommend maintenance therapy with either a longacting muscarinic antagonist (LAMA) or a long-acting beta agonist (LABA) in patients with moderate or severe COPD (Groups B-D) when short-acting muscarinic antagonists (SAMAs) fail to control symptoms and exacerbation rates. ${ }^{2}$ Patients who have persistent symptoms or exacerbations should be treated with a combination of LAMA and LABA (LAMA/LABA) or inhaled corticosteroids (ICS) and LABA (ICS/LABA). To our knowledge, there is no published systematic review that compares all treatment options. The aim of this network meta-analysis was to comprehensively compare the efficacy and safety of the individual agents under the various therapeutic classes of inhalers commonly used in the treatment of COPD, namely SAMAs, LAMAs, LAMA/LABA fixed-dose combinations (FDCs) and ICS/ LABA FDCs. LABA monotherapy was not included in this analysis as it is infrequently used compared to the other classes of inhalers in Singapore.

\section{Materials and methods}

This review followed the PRISMA guidelines.

\section{Search strategy}

A systematic search of PubMed and Embase was conducted up to 15 November 2017. The search strategy employed a combination of medical subject headings and text words related to the drug classes of interest, the term "COPD" and their synonyms (Table S1). Reference lists from published systematic reviews were hand-searched for additional publications. The searches were limited to English language.

\section{Study selection}

Randomized, parallel-group, controlled design studies of $\geq 12$ weeks' duration, which compared LAMA/LABA FDCs (aclidinium/formoterol 400/12 mcg twice a day [AclForm], indacaterol/glycopyrronium $110 / 50 \mathrm{mcg}$ once a day [IndaGlyco], tiotropium/olodaterol $5 / 5 \mathrm{mcg}$ once a day [TioOlo], umeclidinium/vilanterol $62.5 / 25 \mathrm{mcg}$ once a day [UmecVil]), LAMAs (aclidinium $400 \mathrm{mcg}$ once a day
[Acl], glycopyrronium $50 \mathrm{mcg}$ once a day [Glyco], tiotropium $18 \mathrm{mcg}$ [Tio18] or $5 \mathrm{mcg}$ [Tio5] once a day, umeclidinium $62.5 \mathrm{mcg}$ once a day [Umec]), ICS/LABA FDC (fluticasone/ salmeterol 250/50 mcg [SFC250] or 500/50 mcg [SFC500] twice a day, fluticasone/vilanterol $100 / 25 \mathrm{mcg}$ once a day [FFVI], budesonide/formoterol 320/9 mcg twice a day [BudeForm]) and SAMA (ipratropium $40 \mathrm{mcg}$ four times a day [Ipra]) with each other or with placebo were selected if they included adults with stable, moderate-to-very severe COPD. The eligible study treatments were restricted to all combinations at their licensed doses which were available at the time of review. Studies were required to report at least one of the following clinical and health status endpoints: trough $\mathrm{FEV}_{1}$, transitional dyspnea index (TDI), St George's Respiratory Questionnaire (SGRQ) and safety (frequency of adverse events [AEs]). There was considerable heterogeneity in the definition of exacerbation outcomes across the studies, which limited their ability to be pooled in a network. Most of the earlier studies defined exacerbation by the symptoms (eg, at least 3 days of increased sputum production), while more recent studies defined exacerbation by the treatment received (eg, requiring corticosteroids or hospital admission).

\section{Data extraction and risk of bias assessment}

Two authors (MIAA and LET) independently reviewed the search results and assessed the eligibility of the studies for selection. Any disagreements were resolved by discussion to achieve consensus. The data extraction was performed independently using a standard template and checked for discrepancies. Specific data points of interest that were only presented in graphs were extracted using WebPlotDigitizer. ${ }^{3}$ Risk of bias was assessed using the Cochrane Collaboration Risk of Bias tool. ${ }^{4}$ Domains assessed were random sequence generation, allocation concealment, blinding of participants, blinding of outcome assessment, incomplete outcome data and selective outcome reporting. Biases were reported as high or low or unclear. Assumption checking for homogeneity, similarity and consistency was also conducted.

\section{Data synthesis and analysis}

A frequentist random-effects network meta-analysis was performed using the "network" routine within the mvmeta package in Stata 15 statistical software (StataCorp, College Station, TX, USA). ${ }^{5}$ Network meta-analysis allows for simultaneous analysis of direct comparisons of interventions (head-to-head) within randomized controlled trials and indirect comparisons across trials based on a common 
comparator, provided that the studies included are comparable in terms of treatment effect modifiers. The synthesis of direct and indirect evidence produces a more precise and refined estimate of treatment effectiveness by maximizing the use of available data for all treatments within the network. Direct pairwise comparisons were also conducted using the metan package in Stata. ${ }^{6}$

The primary outcome was trough $\mathrm{FEV}_{1}$ (in $\mathrm{mL}$ ); secondary outcomes included were TDI, SGRQ and AEs. For the continuous outcomes $\left(\mathrm{FEV}_{1}, \mathrm{SGRQ}\right.$ and TDI), the mean difference (MD) in the change from baseline values between the two arms was used in the analyses. The minimal clinically important difference (MCID) for $\mathrm{FEV}_{1}$ is $100 \mathrm{~mL}{ }^{7,8}$ The proportions of patients who attained the MCID in TDI (TDI responders, a $\geq 1$ unit increase in TDI) ${ }^{9}$ and SGRQ (SGRQ responders, a $\geq 4$ unit decrease in SGRQ score $)^{10}$ were also analyzed. Observation time points of 12 and 24 weeks were chosen as they were the two consistently reported time points across the studies. A result was considered significant if the $95 \%$ CI did not include 0 or one for the continuous and dichotomous outcomes, respectively. The Surface Under the Cumulative RAnking (SUCRA) curve was obtained to determine the relative probability of a treatment being the best option for each outcome measure. ${ }^{11}$

Possible network inconsistency was assessed using the design-by-treatment model approach described by White. ${ }^{5}$ This approach provided a global test for inconsistency, with a $P$-value $<0.05$ indicating violation of the consistency assumption in the network.

\section{Results}

\section{Search and selection results}

The electronic database search identified 1,611 citations, of which 1,485 were excluded on abstract review (Figure S1). A further 50 were excluded after reviewing the full-text articles, leaving 74 studies reported by 75 articles included in the final selection.

The 74 studies selected for inclusion were published between 2000 and 2017. The study characteristics are presented in Table 1. Thirty-nine studies (53\%) were placebo controlled and 35 were active controlled. Overall, there were more published studies for tiotropium $18 \mathrm{mcg}$ compared to the other agents, given it was the first LAMA licensed for COPD. Most studies (67 studies; 91\%) had large sample sizes with $>200$ participants. All studies included patients aged at least 35 years, with a smoking history of at least 10 packs per year. Six studies only included patients with a prior history of exacerbations, while five studies only included patients without

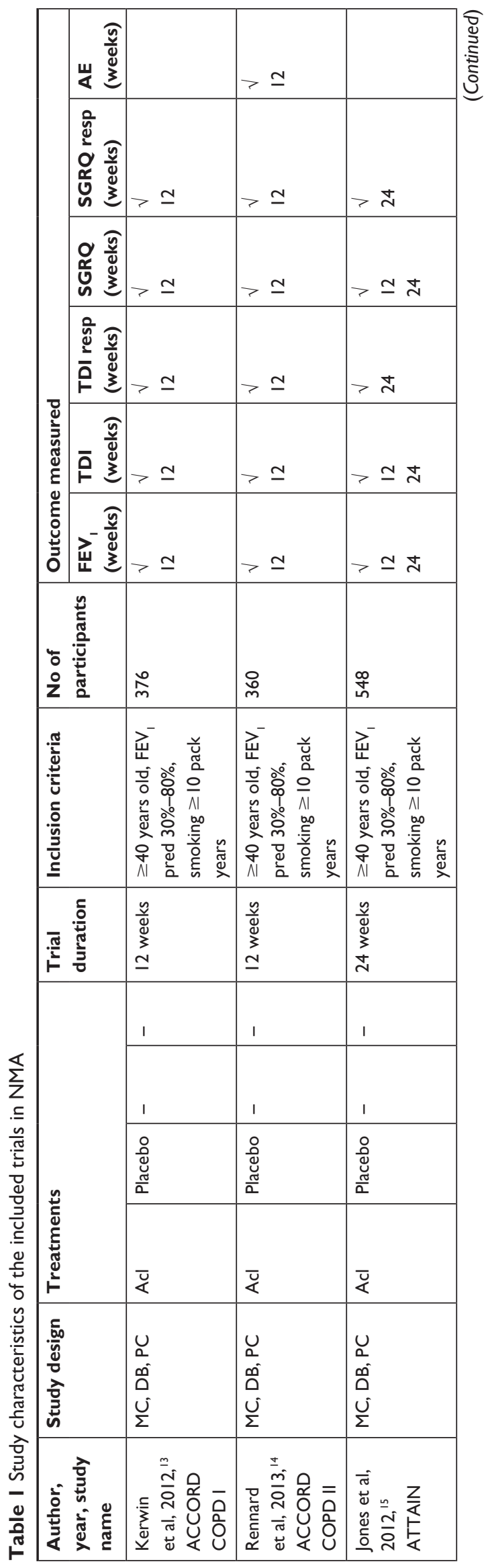




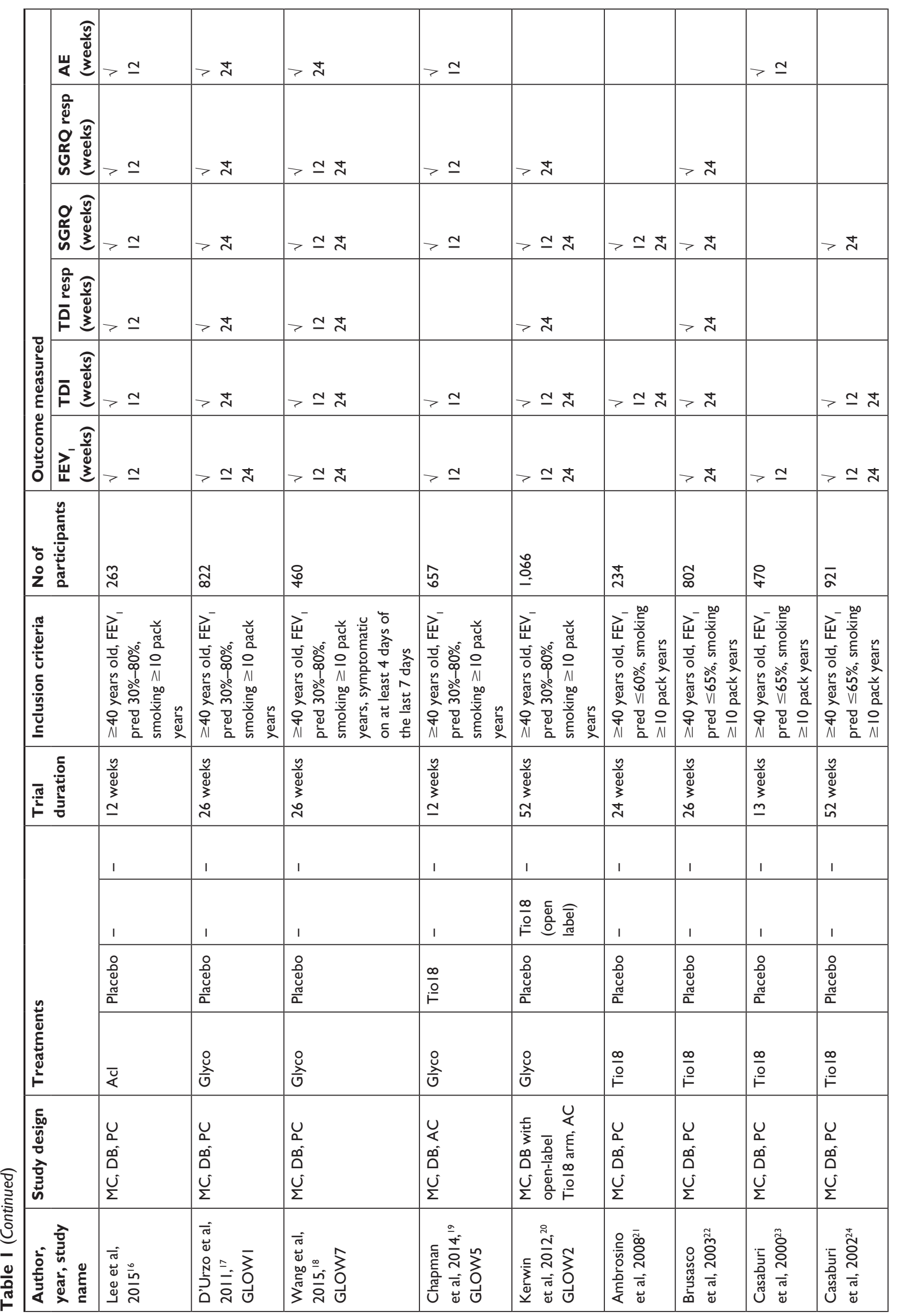




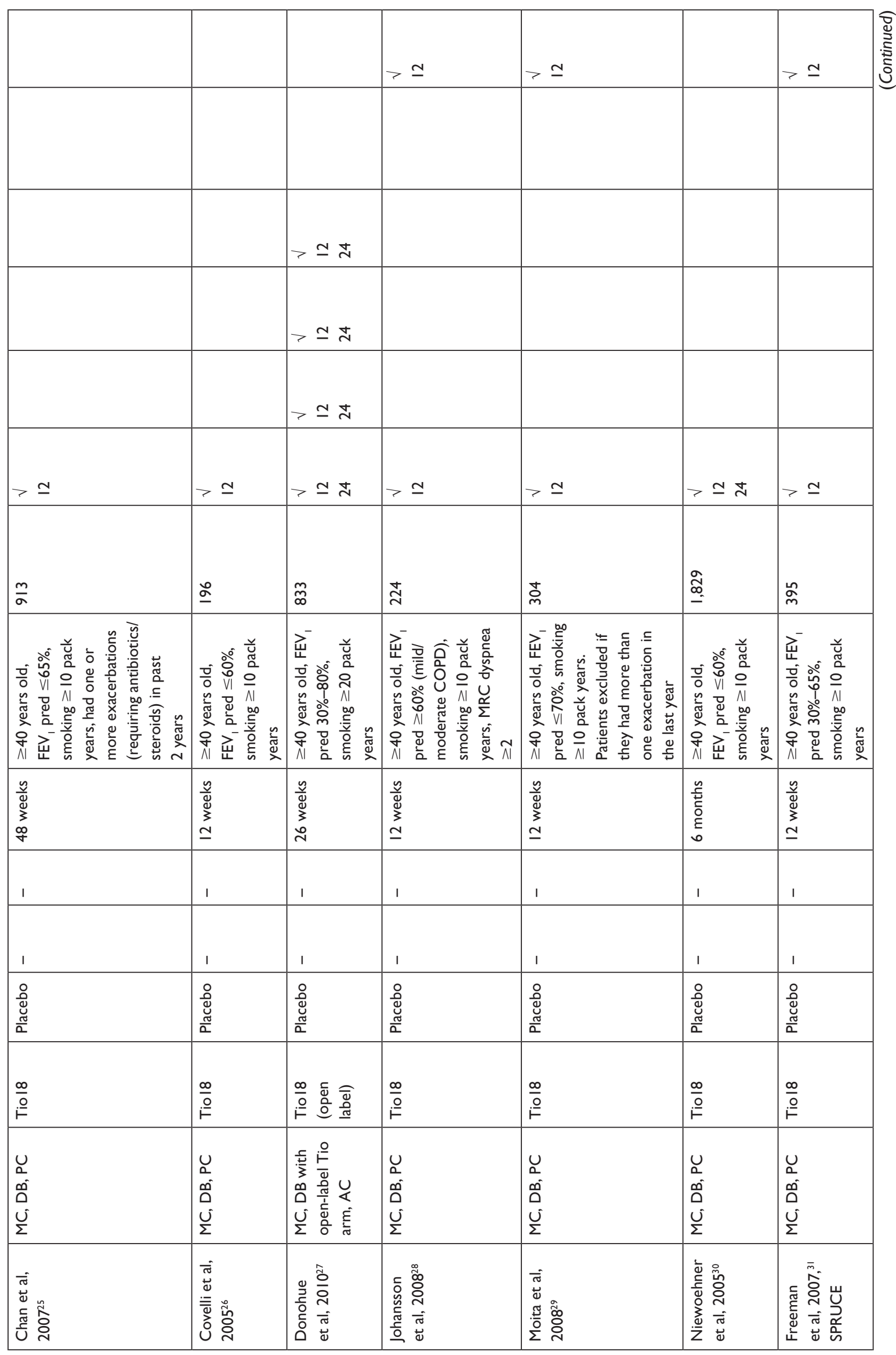




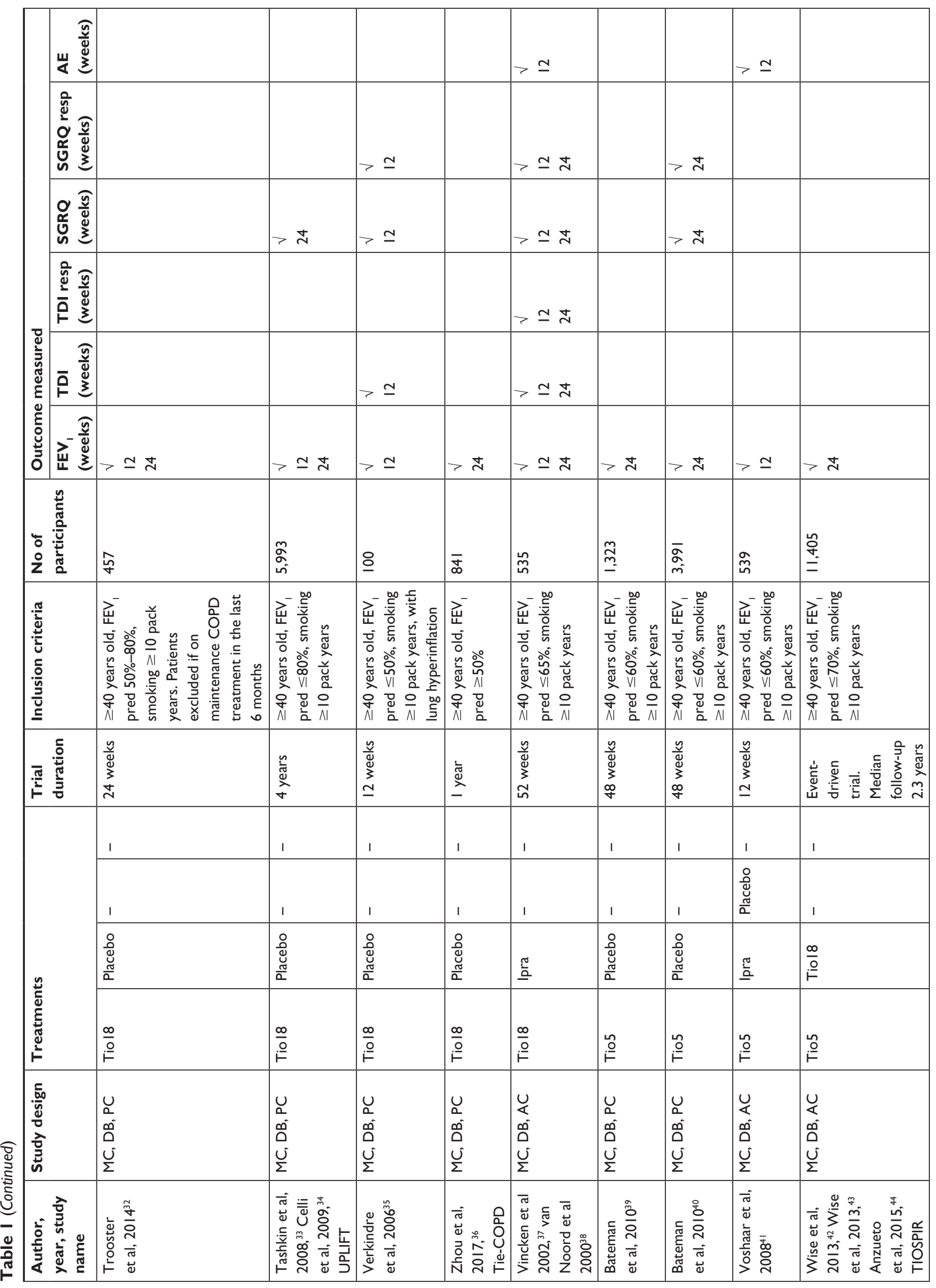




\begin{tabular}{|c|c|c|c|c|c|c|}
\hline$>\simeq$ & $>\simeq$ & $>\simeq$ & & & 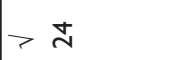 & $> \pm$ \\
\hline & $>\simeq$ & $>\simeq$ & $>\simeq \dot{\sim}$ & & $>d$ & $>d$ \\
\hline$>\simeq$ & $>\simeq$ & $>\simeq$ & $> \pm$ & $> \pm$ & & 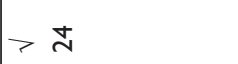 \\
\hline$>\simeq$ & $>\simeq$ & $>\simeq$ & $>\simeq \pm$ & $> \pm$ & $>\mathbb{4}$ & $>A$ \\
\hline$>\simeq$ & $>\simeq$ & $>\simeq$ & $> \pm$ & $> \pm$ & $>d$ & $>\simeq \pm$ \\
\hline$>\simeq$ & $>\simeq$ & $>\simeq$ & $>\simeq \pm$ & $>\simeq \pm$ & & $>\simeq \pm$ \\
\hline$\underline{\underline{m}}$ & 흠 & 芯 & $\stackrel{n}{0}$ & 芯 & ma & 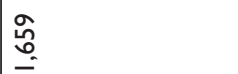 \\
\hline 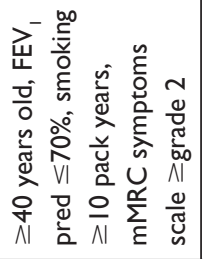 & 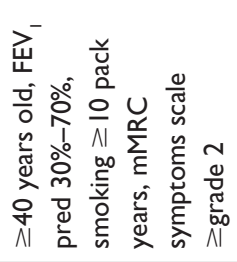 & 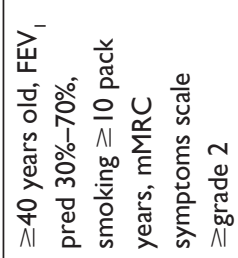 & 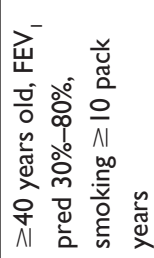 & 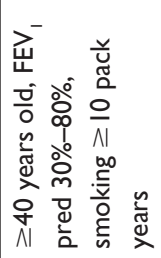 & 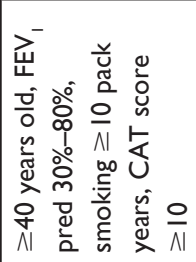 & 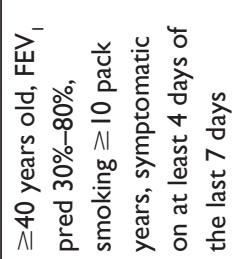 \\
\hline 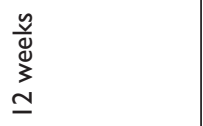 & 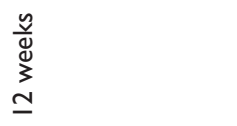 & 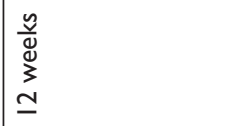 & 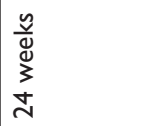 & 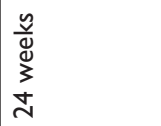 & 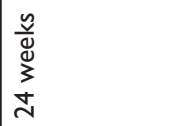 & 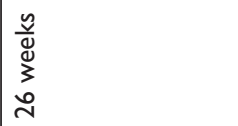 \\
\hline 1 & 1 & 1 & 1 & 1 & 1 & 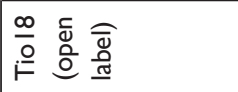 \\
\hline 1 & 1 & 1 & $\begin{array}{l}\circ \\
\stackrel{8}{\tilde{U}} \\
\frac{\tilde{U}}{\alpha} \\
\end{array}$ & $\begin{array}{l}0 \\
\stackrel{8}{0} \\
\frac{\tilde{U}}{\alpha} \\
\frac{\pi}{\alpha}\end{array}$ & 1 & $\begin{array}{l}0 \\
\stackrel{8}{0} \\
\frac{0}{\alpha} \\
\frac{\pi}{\alpha}\end{array}$ \\
\hline 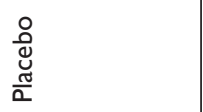 & $\frac{\infty}{\circ}$ & 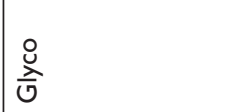 & $\bar{q}$ & $\bar{q}$ & $\begin{array}{l}8 \\
0 \\
0 \\
4 \\
4\end{array}$ & 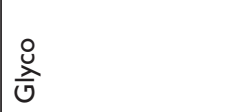 \\
\hline 亗 & 㐫 & 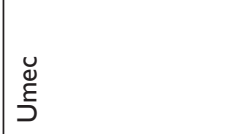 & 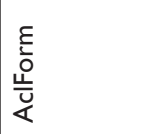 & 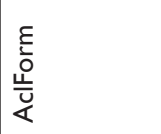 & 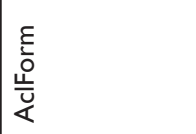 & 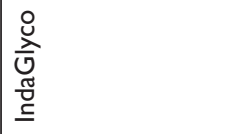 \\
\hline $\begin{array}{l}u \\
\vdots \\
\tilde{\Delta} \\
\dot{u} \\
\dot{v}\end{array}$ & $\begin{array}{l}u \\
\alpha \\
\tilde{\Delta} \\
\dot{u} \\
\dot{v}\end{array}$ & 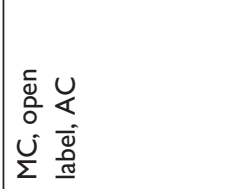 & 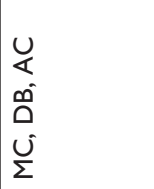 & 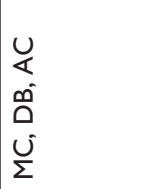 & 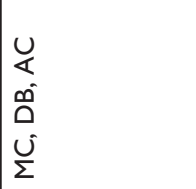 & 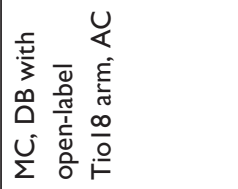 \\
\hline 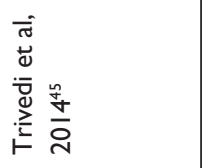 & 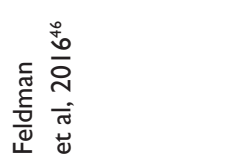 & 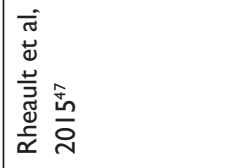 & 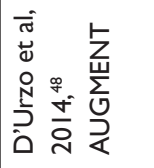 & 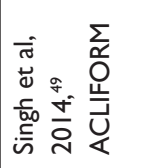 & 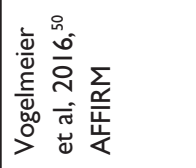 & 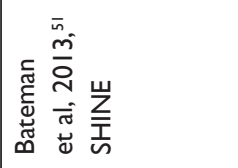 \\
\hline
\end{tabular}




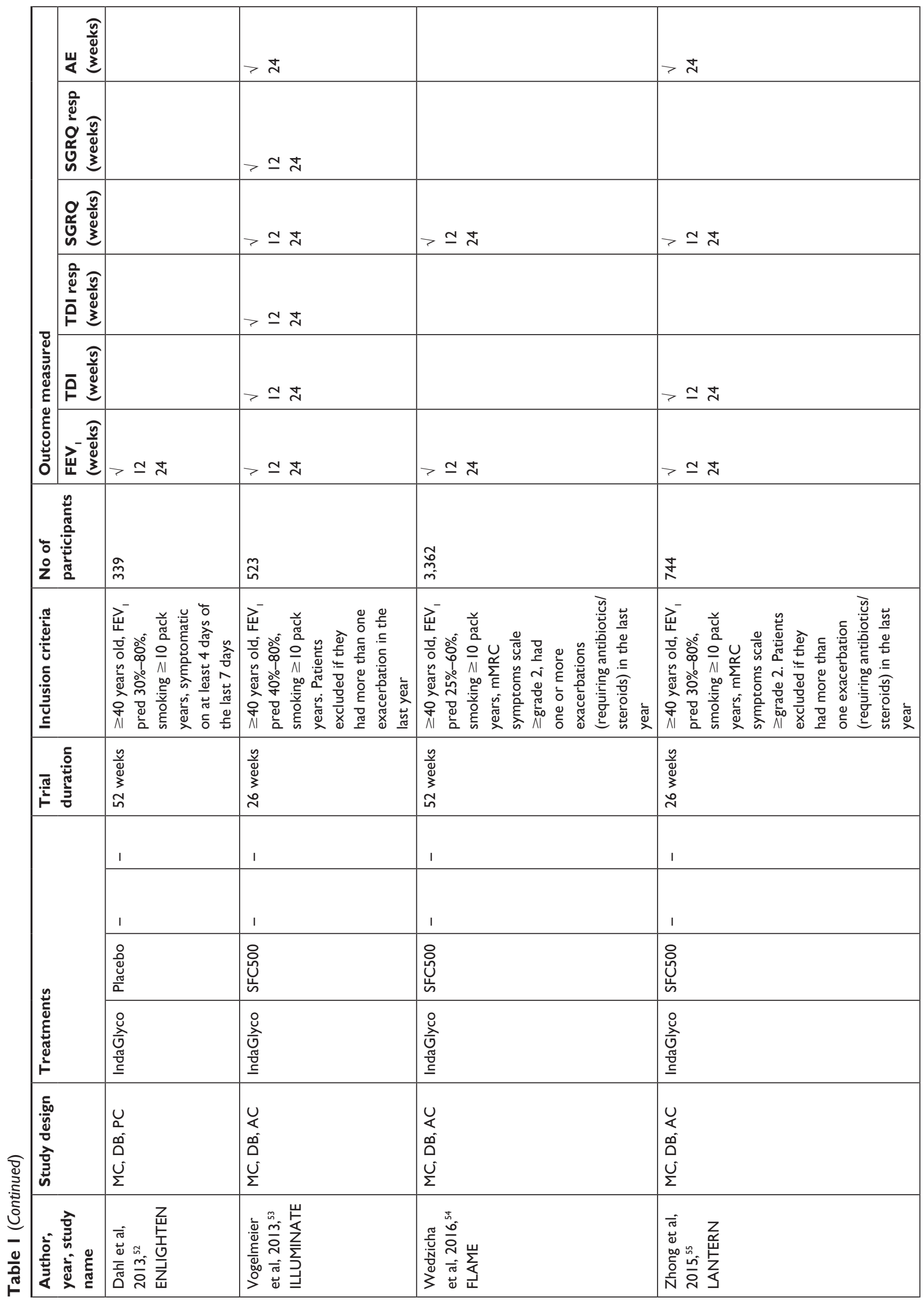




\begin{tabular}{|c|c|c|c|c|c|}
\hline & $>\simeq$ & $>\simeq$ & & & $>\simeq$ \\
\hline$>\simeq \pm$ & $>\simeq$ & $>\simeq$ & 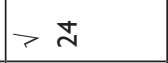 & $> \pm$ & $>\simeq$ \\
\hline$>\simeq \pm$ & $>\simeq$ & $>\simeq$ & $> \pm$ & $>\stackrel{N}{ }$ & $>\simeq$ \\
\hline & $>\simeq$ & $>\simeq$ & & & $>\simeq$ \\
\hline$>\simeq \dot{A}$ & $>\simeq$ & $>\simeq$ & $>\simeq \pm$ & $>\simeq \pm$ & $>\simeq$ \\
\hline 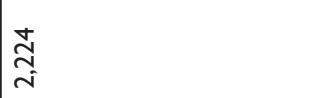 & $\div$ & i̊ & d & $\frac{m}{0}$ & 矣 \\
\hline 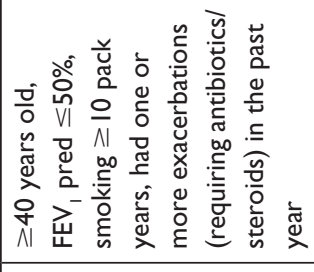 & 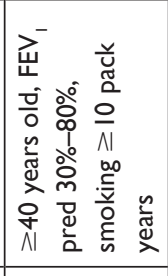 & 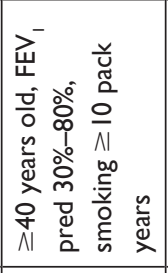 & 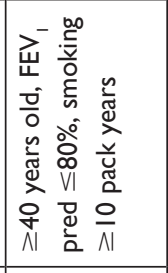 & 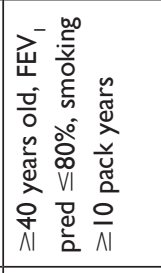 & 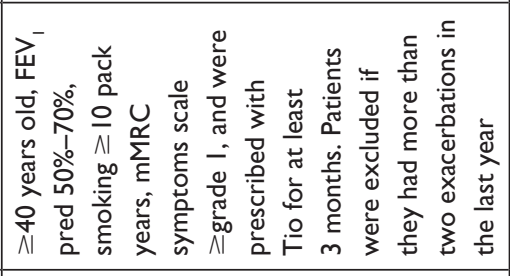 \\
\hline 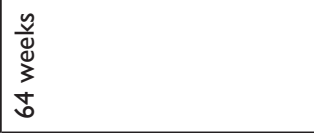 & 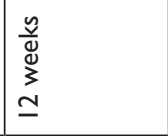 & 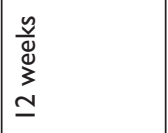 & 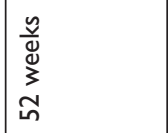 & 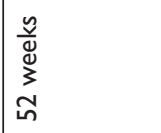 & 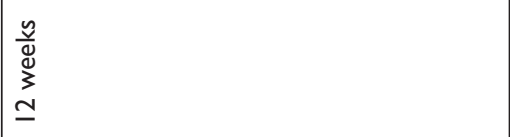 \\
\hline 1 & 1 & 1 & 1 & 1 & 1 \\
\hline 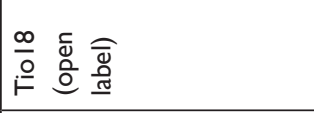 & 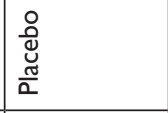 & \begin{tabular}{|l}
0 \\
$\stackrel{0}{0}$ \\
$\frac{\tilde{J}}{\alpha}$ \\
\end{tabular} & 1 & 1 & 1 \\
\hline $\begin{array}{l}\text { O } \\
\text { 츤 }\end{array}$ & $\stackrel{\stackrel{n}{\circ}}{i}$ & $\stackrel{\stackrel{n}{0}}{i=}$ & $\stackrel{\stackrel{n}{\circ}}{\stackrel{\gamma}{F}}$ & $\stackrel{\stackrel{n}{\rho}}{\circ}$ & 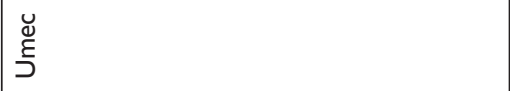 \\
\hline 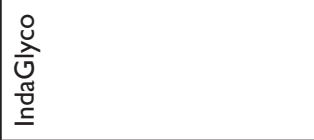 & $\begin{array}{l}\stackrel{\circ}{\circ} \\
\stackrel{\circ}{\circ} \\
i \vdash\end{array}$ & $\begin{array}{l}\stackrel{\circ}{O} \\
\stackrel{\circ}{\circ} \\
i=\end{array}$ & $\begin{array}{l}\stackrel{\circ}{O} \\
\stackrel{O}{\circ} \\
i \vdash\end{array}$ & $\begin{array}{l}\stackrel{\circ}{O} \\
\stackrel{\circ}{\circ} \\
i=\end{array}$ & \begin{tabular}{|l}
$\overline{\bar{z}}$ \\
$\overline{\tilde{u}}$ \\
$\bar{J}$
\end{tabular} \\
\hline 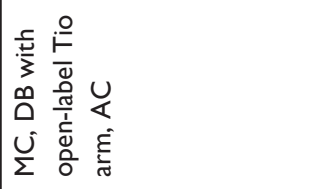 & 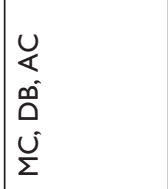 & $\begin{array}{l}u \\
\Psi \\
0 \\
0 \\
u \\
\dot{u}\end{array}$ & 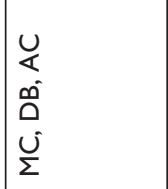 & 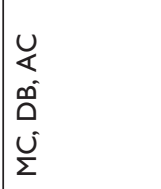 & 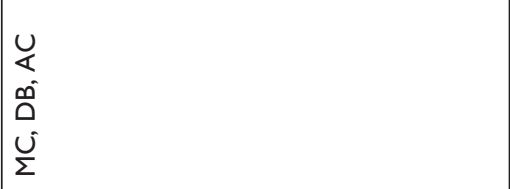 \\
\hline 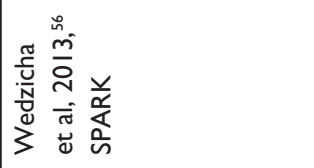 & 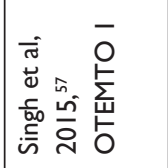 & 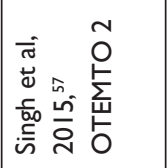 & 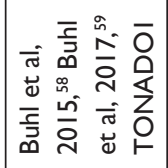 & 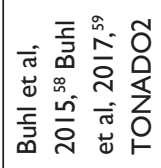 & 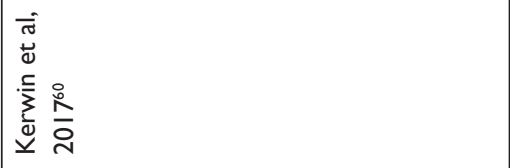 \\
\hline
\end{tabular}




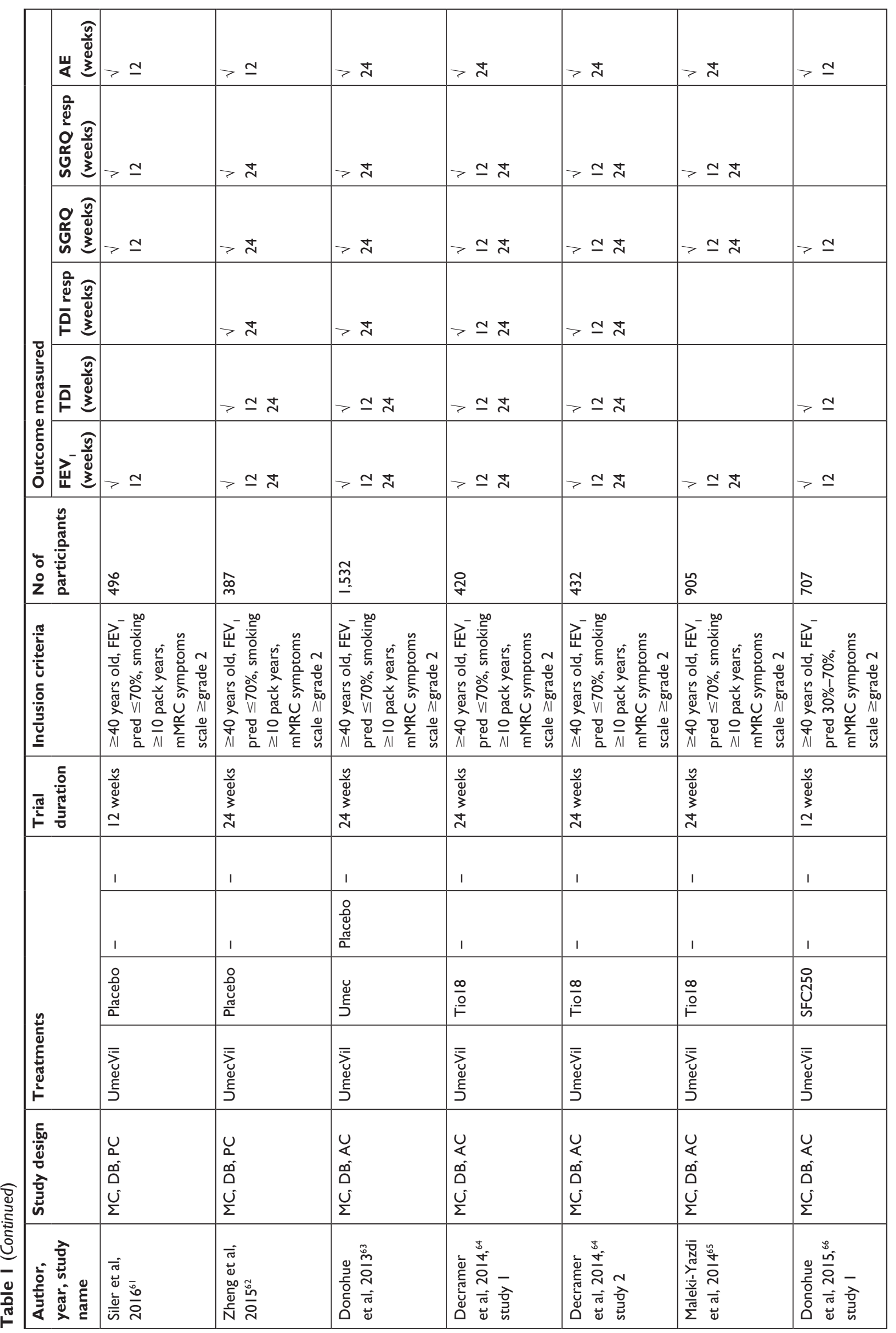




\begin{tabular}{|c|c|c|c|c|}
\hline$>\simeq$ & $>\simeq$ & 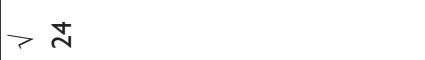 & & \\
\hline & $>\simeq$ & $> \pm$ & & \\
\hline$>\simeq$ & $>\simeq$ & $> \pm$ & & $>\simeq \pm$ \\
\hline$>\simeq$ & $>\simeq$ & & & \\
\hline$>\simeq$ & $>\simeq$ & $> \pm$ & $> \pm$ & $>\simeq \pm$ \\
\hline 尺 & $\frac{0}{N}$ & $\hat{\text { in }}$ & 占 & $\stackrel{2}{\text { N }}$ \\
\hline 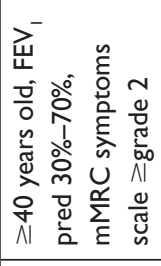 & 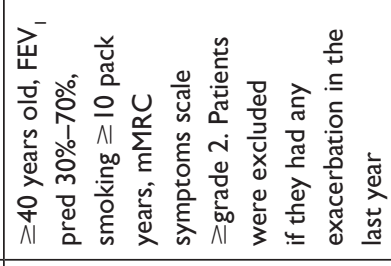 & 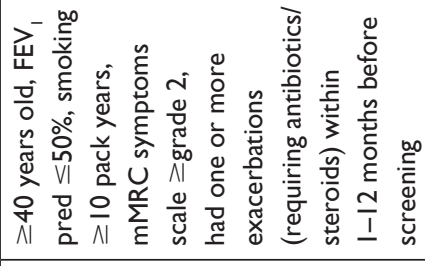 & 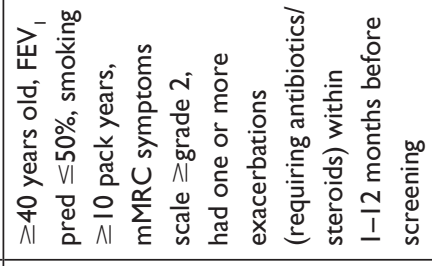 & 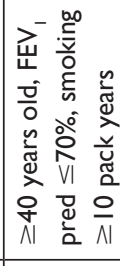 \\
\hline 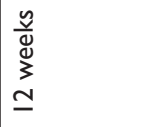 & 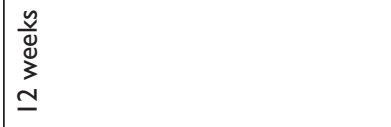 & 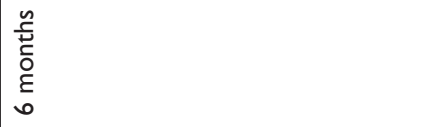 & 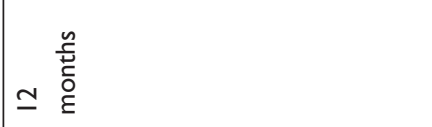 & 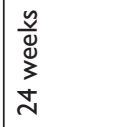 \\
\hline 1 & 1 & 1 & 1 & 1 \\
\hline 1 & 1 & 1 & 1 & 1 \\
\hline $\begin{array}{l}0 \\
\text { ஸे } \\
\text { U్ } \\
\omega\end{array}$ & 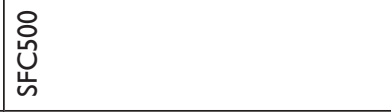 & $\begin{array}{l}\circ \\
\stackrel{8}{\mathscr{U}} \\
\frac{\pi}{\alpha} \\
\frac{\pi}{\alpha}\end{array}$ & $\begin{array}{l}\circ \\
\stackrel{8}{\ddot{U}} \\
\frac{\tilde{\sigma}}{\alpha} \\
\end{array}$ & $\begin{array}{l}\circ \\
\stackrel{8}{\mathscr{Q}} \\
\frac{\tilde{\sigma}}{\alpha} \\
\end{array}$ \\
\hline $\begin{array}{l}\overline{\bar{\nu}} \\
\stackrel{\tilde{u}}{\xi} \\
\bar{\nu}\end{array}$ & $\begin{array}{l}\overline{\bar{z}} \\
\tilde{\tilde{u}} \\
\bar{\nu}\end{array}$ & 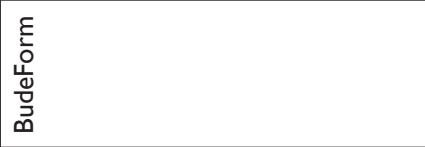 & 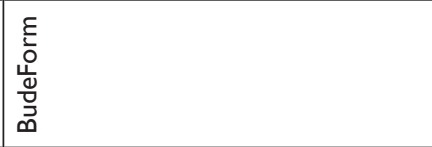 & 站 \\
\hline 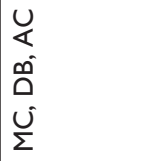 & 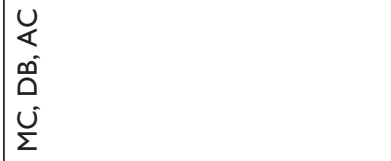 & $\begin{array}{l}u \\
0 \\
0 \tilde{0} \\
u \\
\dot{u}\end{array}$ & $\begin{array}{l}u \\
0 \\
\text { صิ } \\
u \\
\dot{u}\end{array}$ & 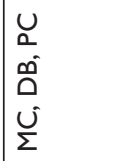 \\
\hline 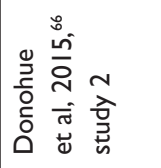 & 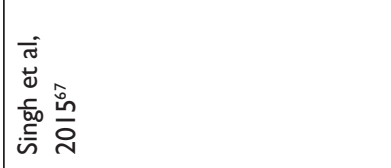 & 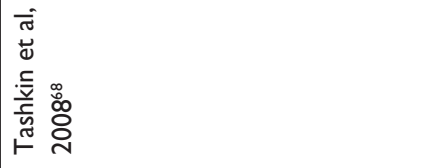 & 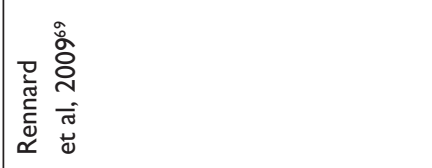 & 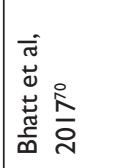 \\
\hline
\end{tabular}




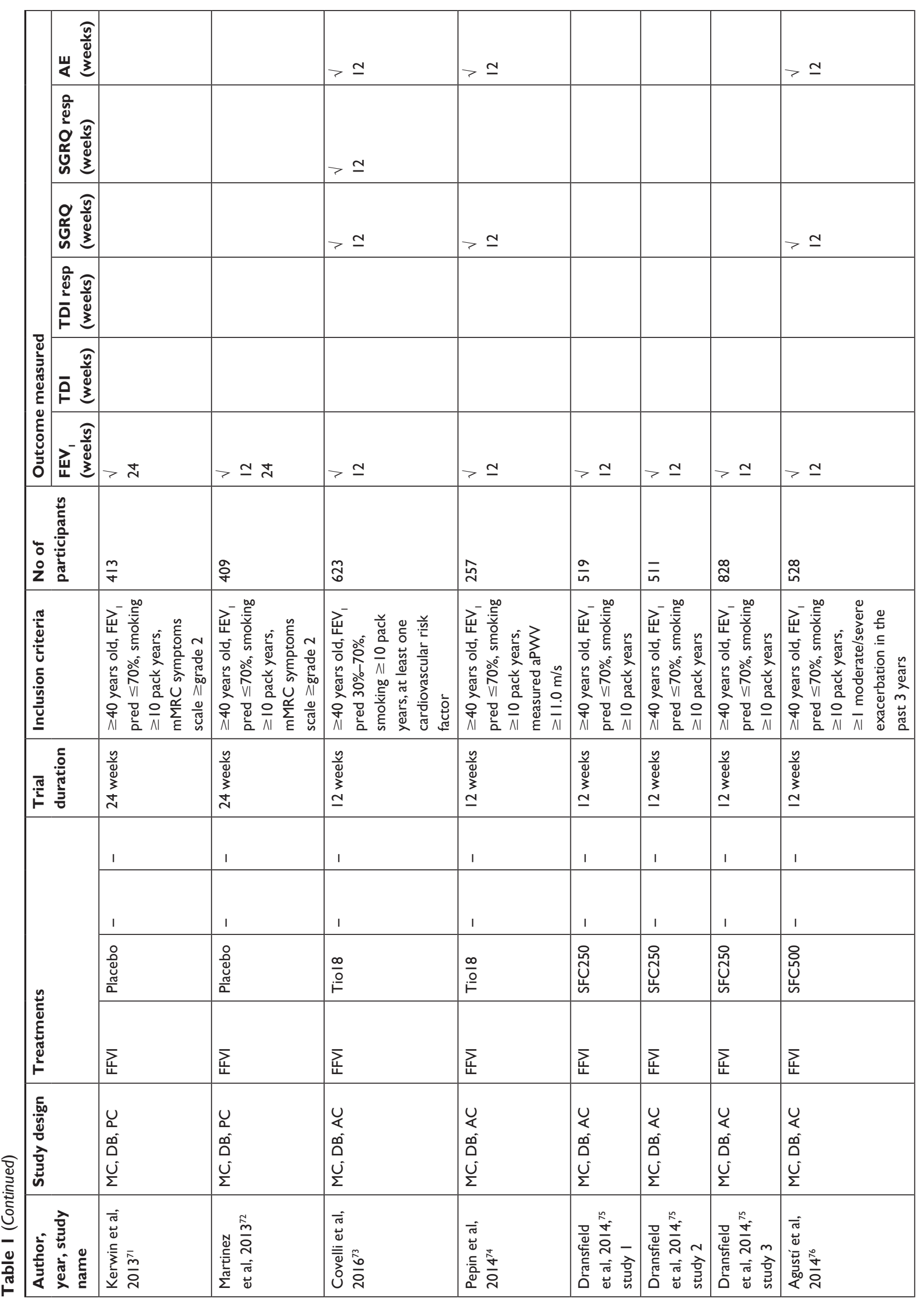




\begin{tabular}{|c|c|c|c|c|c|c|c|c|c|}
\hline$>\simeq$ & & $> \pm$ & & $> \pm$ & $>\simeq$ & & $>\simeq$ & $>\simeq$ & खे \\
\hline & & & $>\underset{4}{ }$ & $> \pm$ & & $>\simeq$ & $>\simeq$ & & 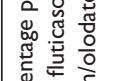 \\
\hline$>\simeq$ & $>\simeq \pm$ & $>\simeq \pm$ & $>\stackrel{ \pm}{4}$ & $>\stackrel{ \pm}{\Lambda}$ & $>\simeq$ & $>\simeq$ & & & $\because \ddot{x}$ \\
\hline i̊ & 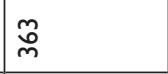 & 岕 & $\frac{9}{0}$ & 年 & ㅇ & $\hat{6}$ & d & $\underline{\infty}$ & $\frac{5}{n} \frac{\partial}{a}$ \\
\hline 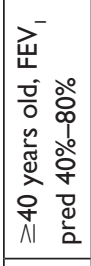 & 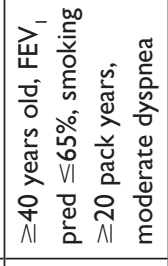 & 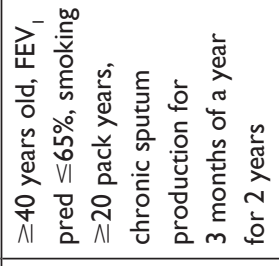 & 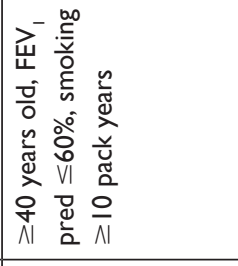 & 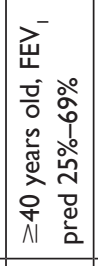 & 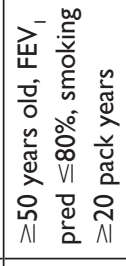 & 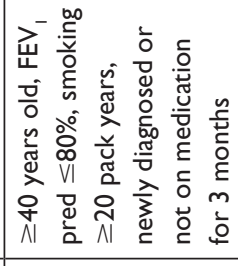 & 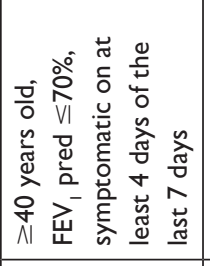 & 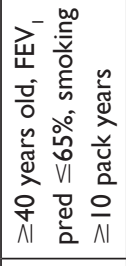 & 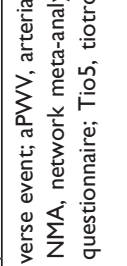 \\
\hline 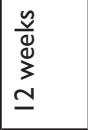 & 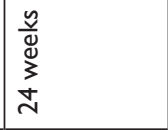 & 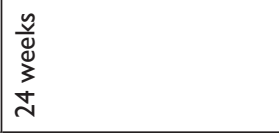 & 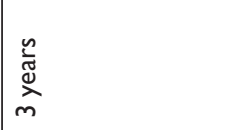 & 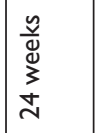 & 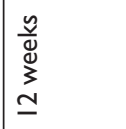 & 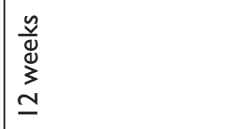 & 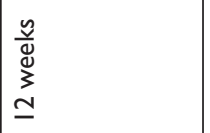 & 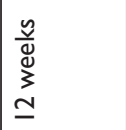 & 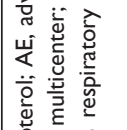 \\
\hline 1 & 1 & 1 & 1 & 1 & 1 & 1 & 1 & 1 & \\
\hline 1 & 1 & 1 & 1 & 1 & 1 & 1 & 1 & 1 & \\
\hline 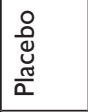 & \begin{tabular}{|l}
$\circ$ \\
$\stackrel{8}{\ddot{U}}$ \\
$\frac{\pi}{0}$ \\
0
\end{tabular} & $\begin{array}{l}0 \\
\stackrel{8}{\mathscr{U}} \\
\frac{\tilde{U}}{\alpha} \\
\end{array}$ & $\begin{array}{l}\circ \\
\stackrel{8}{\mathscr{U}} \\
\frac{\tilde{\sigma}}{\alpha} \\
\end{array}$ & 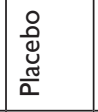 & $\stackrel{\infty}{\stackrel{\infty}{\circ}}$ & $\frac{\infty}{\stackrel{\circ}{\circ}}$ & 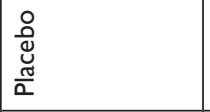 & 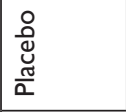 & 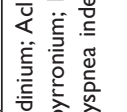 \\
\hline 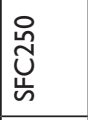 & 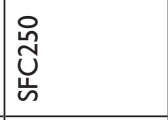 & $\begin{array}{l}8 \\
0 \\
0 \\
4 \\
4 \\
\end{array}$ & $\begin{array}{l}8 \\
0 \\
0 \\
4 \\
4 \\
\end{array}$ & $\begin{array}{l}8 \\
0 \\
0 \\
4 \\
\text { w } \\
\end{array}$ & $\begin{array}{l}8 \\
0 \\
0 \\
4 \\
4 \\
\end{array}$ & $\begin{array}{l}8 \\
0 \\
0 \\
4 \\
w \\
\end{array}$ & 兽 & 兽 & 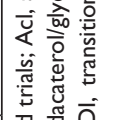 \\
\hline $\begin{array}{l}u \\
0 \\
\infty \\
0 \\
u \\
\Sigma\end{array}$ & $\begin{array}{l}u \\
0 \\
\infty \\
0 \\
u \\
\dot{\Sigma}\end{array}$ & $\begin{array}{l}u \\
0 \\
\infty \\
0 \\
u \\
\Sigma \\
\Sigma\end{array}$ & $\begin{array}{l}u \\
0 \\
\infty \\
0 \\
u \\
\dot{v}\end{array}$ & \begin{tabular}{|l}
$u$ \\
0 \\
$\infty$ \\
0 \\
$u$ \\
$\vdots$ \\
\end{tabular} & 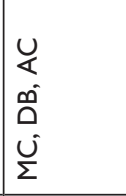 & 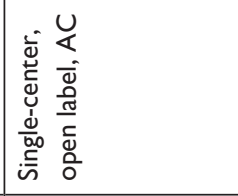 & $\begin{array}{l}u \\
0 \\
\infty \\
0 \\
u \\
\dot{v}\end{array}$ & $\begin{array}{l}u \\
0 \\
\infty \\
0 \\
u \\
\dot{v}\end{array}$ & 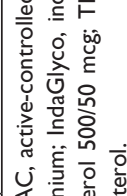 \\
\hline 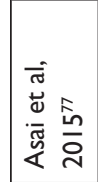 & 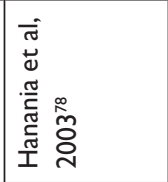 & 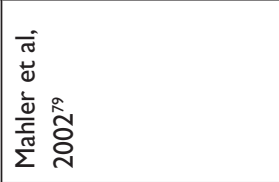 & 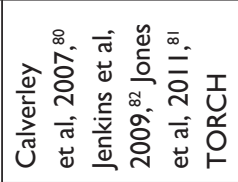 & 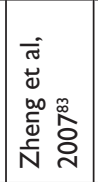 & 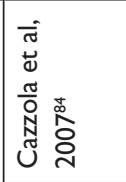 & 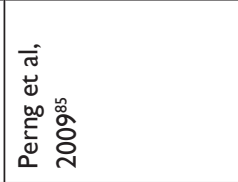 & 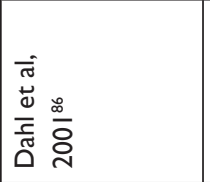 & 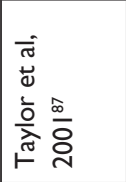 & 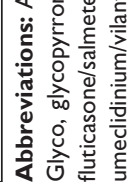 \\
\hline
\end{tabular}


any exacerbation history. The remaining 63 studies did not specify exacerbation history in their inclusion/exclusion criteria. The most commonly reported primary outcome across the studies was lung function (trough $\mathrm{FEV}_{1}$ ); limited data for other patient-related outcomes (TDI and SGRQ) were also available. Data from the intention-to-treat and full analysis set for all trials were extracted. The network plots for trough $\mathrm{FEV}_{1}$ at weeks 12 and 24 are shown in Figure 1.

A total of 74,832 patients were included in the 74 studies. The key patient characteristics and assessment of risk of bias for each study are presented in Table 2. Mean ages ranged from 61 to 73 years; proportion of males and current smokers ranged from $48 \%$ to $99 \%$ and from $22 \%$ to $88 \%$, respectively. The mean $\mathrm{FEV}_{1}$ predicted at baseline ranged from $35 \%$ to $78 \%$. Majority of the studies were assessed to have low or unclear risk of bias. Inconsistency models were not significant for all outcomes, implying that the consistency assumption was not violated.

\section{Efficacy}

\section{Trough FEV , change from baseline}

Trough FEV 1 results were reported in 59 studies at week 12 and in 39 studies at week 24. All LAMAs, LAMA/LABAs and ICS/LABAs led to significantly greater improvement in trough $\mathrm{FEV}_{1}$ compared to SAMA and placebo at weeks 12 and 24 (Tables 3 and 4). While some of the comparisons among LAMAs, LAMA/LABAs and ICS/LABAs showed statistical significance, the results were generally not clinically significant with respect to an MCID of $100 \mathrm{~mL}$. For example, among the LAMAs, Umec led to statistically significant improvement in trough $\mathrm{FEV}_{1}$ at week $12 \mathrm{com}$ pared to Tio18 (mean difference [MD] of $37 \mathrm{~mL}, 95 \% \mathrm{CI}$ 13-62 mL) and Glyco (MD $31 \mathrm{~mL}, 95 \%$ CI 6-57 mL). However, there were no significant differences in trough $\mathrm{FEV}_{1}$ for all LAMA vs LAMA comparisons at week 24. Among the LAMA/LABAs, there were no significant differences between IndaGlyco, TioOlo and UmecVil at weeks 12 and 24. IndaGlyco and UmecVil showed statistically significant improvement in $\mathrm{FEV}_{1}$ when compared to AclForm at both weeks 12 and 24. The MDs ranged from 43 to $59 \mathrm{~mL}$ (point estimates). Statistically significant improvement at weeks 12 and 24 was also seen for IndaGlyco, TioOlo and UmecVil compared to all LAMAs. The MDs ranged from 48 to $88 \mathrm{~mL}$ (point estimates). On the other hand, AclForm exhibited no significant difference compared to any LAMAs. Among the ICS/LABAs, the results were mixed with some showing significant differences between the agents (FFVI vs SFC250 at week 12, MD $40 \mathrm{~mL}$; FFVI vs BudeForm at week 24, MD 58 $\mathrm{mL}$; BudeForm vs SFC250 at week 24, MD $-82 \mathrm{~mL}$ ). When compared to the LAMAs, ICS/LABAs had a similar effect on $\mathrm{FEV}_{1}$ at week 12, except SFC250 vs Umec (MD $-52 \mathrm{~mL}$, 95\% CI -85 to $-18 \mathrm{~mL}$, favoring Umec) and FFVI vs Tio 18 (MD $25 \mathrm{~mL}, 95 \%$ CI 4-47 mL, favoring FFVI). At week 24, ICS/LABAs were generally comparable to the LAMAs, except BudeForm vs Glyco (MD $-40 \mathrm{~mL}, 95 \% \mathrm{CI}-77$ to $3 \mathrm{~mL}$, favoring Glyco) and Tio 18 (MD $-35 \mathrm{~mL}, 95 \% \mathrm{CI}-69$
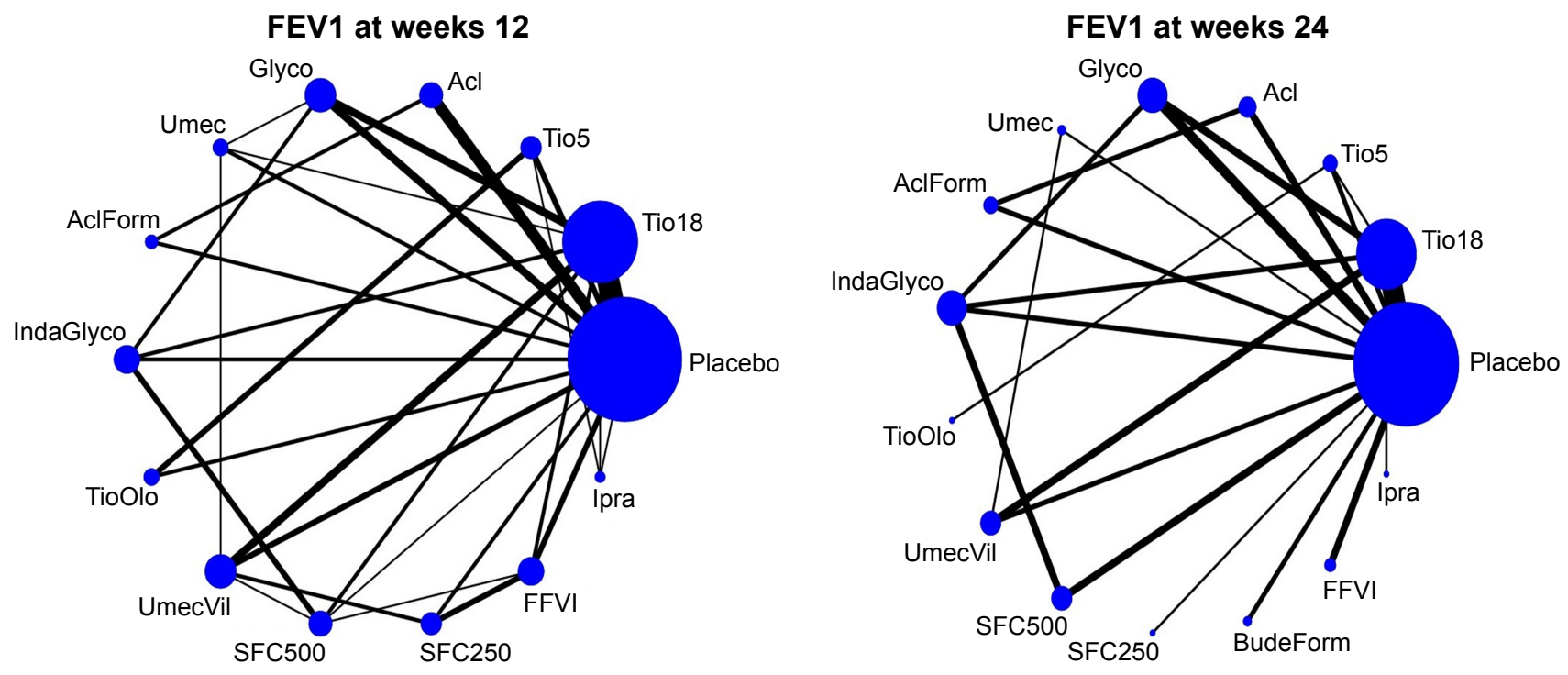

Figure I Evidence network of available trials showing direct comparisons of agents with respect to lung function (trough FEV) at weeks I2 and 24 .

Note: The size of each treatment node is weighted by the number of studies.

Abbreviations: Acl, aclidinium; AclForm, aclidinium/formoterol; BudeForm, budesonide/formoterol; FFVI, fluticasone/vilanterol; Glyco, glycopyrronium; IndaGlyco, indacaterol/glycopyrronium; Ipra, ipratropium; SFC250, fluticasone/salmeterol 250/50 mcg; SFC500, fluticasone/salmeterol 500/50 mcg; Tio5, tiotropium 5 mcg; Tiol8, tiotropium 18 mcg; TioOlo, tiotropium/olodaterol; Umec, umeclidinium; UmecVil, umeclidinium/vilanterol. 


\begin{tabular}{|c|c|c|c|c|c|c|c|c|c|c|c|c|c|}
\hline 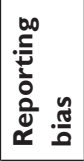 & 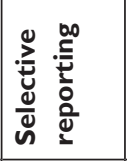 & 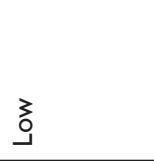 & o & 岁 & $3_{0}^{3}$ & 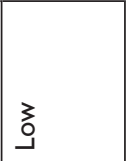 & 岁 & 宐 & 3 & 3. & 苞 & 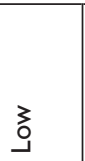 & 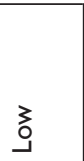 \\
\hline 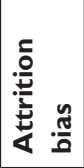 & 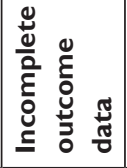 & 光 & o & 䀜 & o & o & 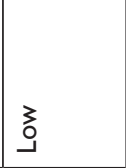 & 盛 & 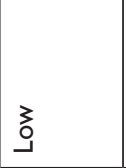 & ${ }_{0}^{3}$ & $\begin{array}{l}\text { 嘉 } \\
\underline{\underline{I}}\end{array}$ & ?3. & 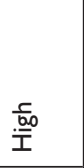 \\
\hline 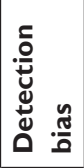 & 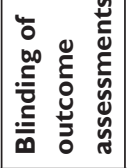 & 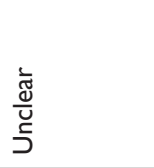 & 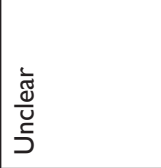 & 弪 & 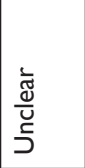 & $\begin{array}{l}\frac{c}{600} \\
\frac{.00}{I}\end{array}$ & 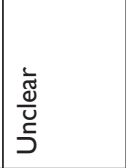 & 光 & 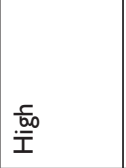 & ${ }_{0}^{3}$ & 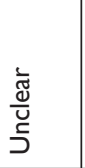 & 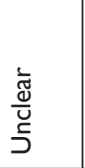 & 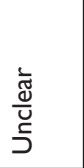 \\
\hline 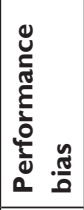 & 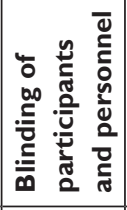 & 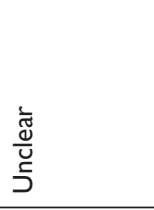 & 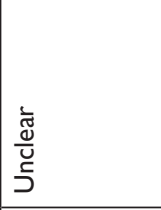 & 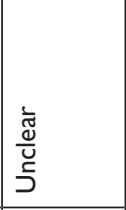 & 夰 & 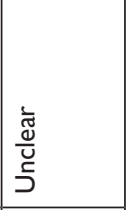 & 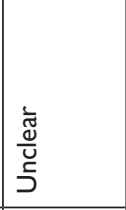 & 3 & $\begin{array}{l}\text { 亳 } \\
\underline{\underline{\underline{I}}}\end{array}$ & $\stackrel{3}{3}$ & 3. & 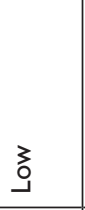 & $\underbrace{3}_{0}$ \\
\hline$n$ & 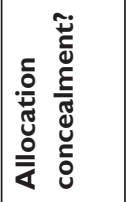 & 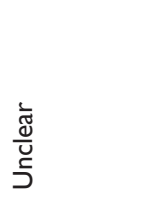 & 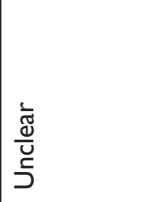 & 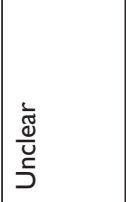 & 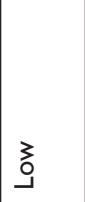 & 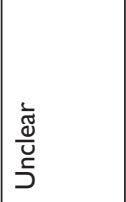 & 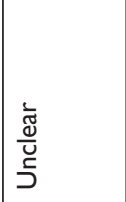 & 告 & 艿 & 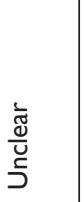 & $\begin{array}{l}\frac{\grave{d}}{\bar{J}} \\
\frac{\mathrm{J}}{5}\end{array}$ & $\begin{array}{l}\frac{\grave{d}}{\frac{d}{\breve{~}}} \\
\text { J }\end{array}$ & 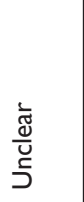 \\
\hline 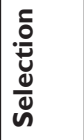 & 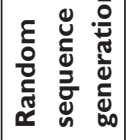 & 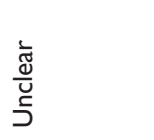 & 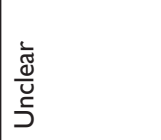 & 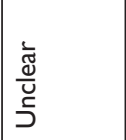 & 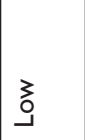 & 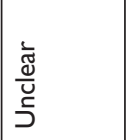 & 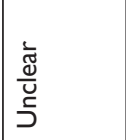 & 光 & 告 & 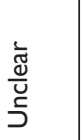 & $\begin{array}{l}\frac{\grave{d}}{d} \\
\frac{d}{\breve{J}}\end{array}$ & 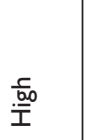 & 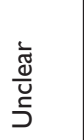 \\
\hline 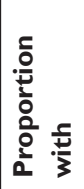 & 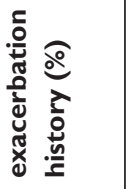 & $\frac{\mathscr{r}}{z}$ & 号 & 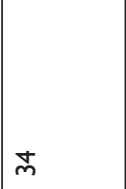 & 号 & $\frac{\stackrel{i}{N}}{\text { N }}$ & $\begin{array}{l}\stackrel{R}{\sim} \\
\sim \\
\sim\end{array}$ & $\begin{array}{l}\stackrel{8}{0} \\
\stackrel{\sim}{\sim}\end{array}$ & $\begin{array}{l}\text { h̊ } \\
\text { di }\end{array}$ & 旁 & 号 & 号 & 号 \\
\hline 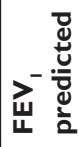 & & ثे & กิ & 吕 & กิ & 䓟 & $\bar{n}$ & 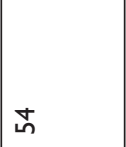 & เீ & $\bar{\sigma}$ & j్ & के & 㝏 \\
\hline 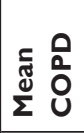 & 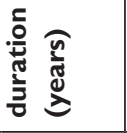 & 兴 & 兰 & 孚 & 号 & సె. & $\stackrel{+}{*}$ & య & $\stackrel{\circ}{\circ}$ & $\bar{\Xi}$ & ڤ̆ & $\therefore$ & $\stackrel{+}{\infty}$ \\
\hline 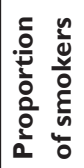 & & J & n̊ & 苟 & 号 & 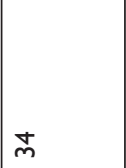 & $\pi$ & 의 & เุ & 兑 & 足 & 号 & $\stackrel{o}{Z}$ \\
\hline 胥 & & นุ & ชิ & ชู & $\stackrel{\infty}{\infty}$ & पे & นึ & กิ & छे & $\widehat{6}$ & ఫే & น & นึ \\
\hline 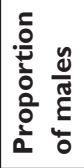 & & กี & ผ & $\stackrel{\infty}{\infty}$ & 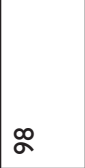 & $\infty$ & 2 & N & t & ১ & 尺 & นิ & น \\
\hline 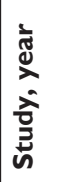 & & 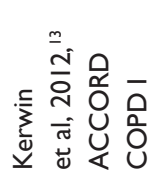 & 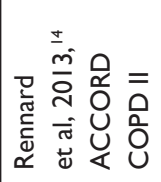 & 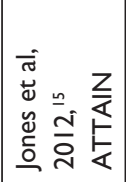 & 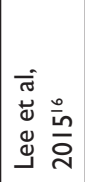 & 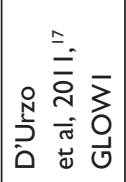 & 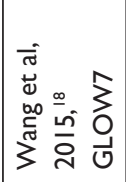 & 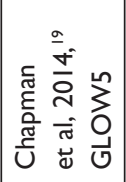 & 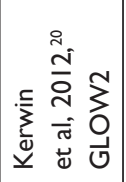 & 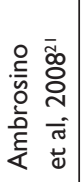 & 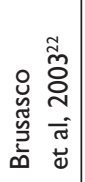 & 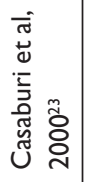 & 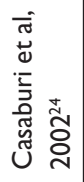 \\
\hline
\end{tabular}




\begin{tabular}{|c|c|c|c|c|c|c|c|c|c|c|c|c|c|c|c|}
\hline 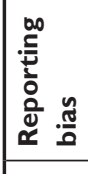 & 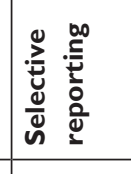 & 3. & 3 & $\stackrel{3}{9}$ & $\stackrel{3}{9}$ & 3 & $\stackrel{3}{9}$ & 3 & 3 & 3 & $\stackrel{3}{9}$ & $\stackrel{3}{9}$ & 3 & $\stackrel{3}{9}$ & 当 \\
\hline 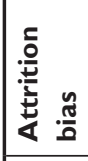 & 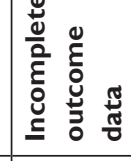 & 产施 & 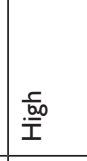 & \begin{tabular}{|l} 
亳 \\
I.
\end{tabular} & 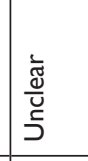 & 3 & \begin{tabular}{|l}
$\frac{5}{b_{0}}$ \\
$I$
\end{tabular} & $\begin{array}{l}\frac{5}{\underline{\underline{b o}}} \\
\underline{I}\end{array}$ & 3 & 3 & 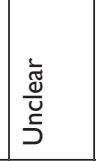 & 3 & $\begin{array}{l}\frac{b}{\underline{b}} \\
\underline{I}\end{array}$ & \begin{tabular}{|l}
$\frac{\underline{\underline{b}}}{\bar{I}}$ \\
\end{tabular} & 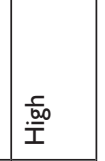 \\
\hline 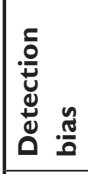 & 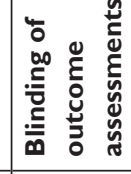 & 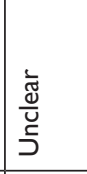 & \begin{tabular}{|l}
$\frac{\bar{w}}{\bar{g}}$ \\
$\frac{5}{5}$
\end{tabular} & $\mid \frac{\underline{\underline{b}}}{\underline{\underline{I}}}$ & 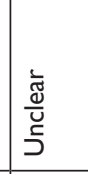 & $\begin{array}{l}\frac{\bar{g}}{\bar{g}} \\
5 \\
5\end{array}$ & 3 & 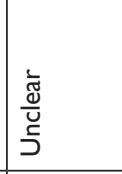 & $\begin{array}{l}\frac{5}{\bar{g}} \\
\frac{u}{5} \\
\end{array}$ & 3 & \begin{tabular}{|l}
$\frac{5}{\bar{g}}$ \\
$\frac{\vec{g}}{5}$
\end{tabular} & 音 & \begin{tabular}{|l}
$\frac{5}{\bar{g}}$ \\
$\frac{5}{5}$
\end{tabular} & \begin{tabular}{|l|l}
$\frac{\bar{d}}{\tilde{J}}$ \\
$\frac{\tilde{J}}{5}$ \\
\end{tabular} & 当 \\
\hline 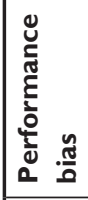 & 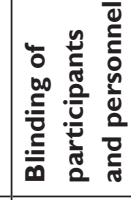 & 3 & \begin{tabular}{|l}
$\frac{\overline{\mathrm{g}}}{\mathrm{u}}$ \\
$\frac{5}{5}$
\end{tabular} & $\mid \frac{\underline{\underline{\underline{b}}}}{\overline{\underline{y}}}$ & $3_{0}^{3}$ & s & 3 & 30 & 3 & క3 & ? & 岁 & 告 & $3_{0}^{3}$ & 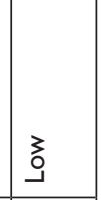 \\
\hline . & 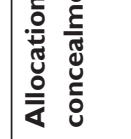 & 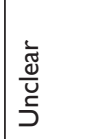 & 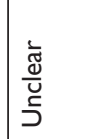 & 暍 & 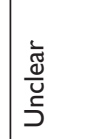 & 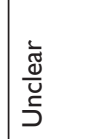 & 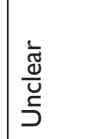 & 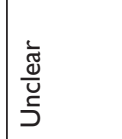 & 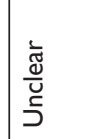 & 3 & 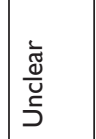 & 䚄 & 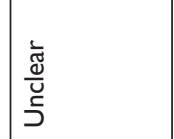 & 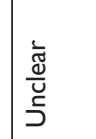 & 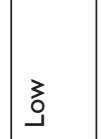 \\
\hline & 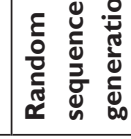 & 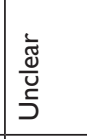 & 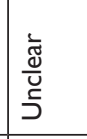 & 3. & 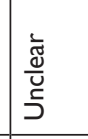 & 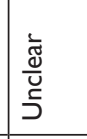 & 3 & 3 & 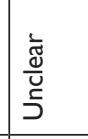 & 3 & 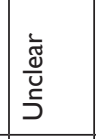 & 3 & 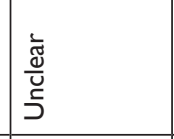 & 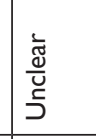 & 3 \\
\hline 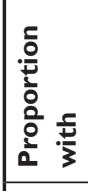 & 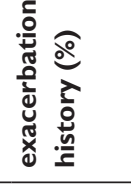 & 음 & $\frac{\alpha}{z}$ & $\frac{\alpha}{z}$ & 金 & $\stackrel{\alpha}{z}$ & $\frac{\tilde{\alpha}}{z}$ & $\frac{\alpha}{z}$ & $\frac{\alpha}{z}$ & $\frac{\alpha}{z}$ & $\frac{\alpha}{z}$ & $\frac{\tilde{\alpha}}{z}$ & 足 & $\frac{\alpha}{z}$ & 足 \\
\hline 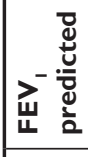 & & ळ & m & in & $\Re$ & $\bar{\gamma}$ & $\stackrel{\infty}{\circ}$ & f & $\stackrel{\circ}{\circ}$ & $\underset{\gamma}{\infty}$ & $\stackrel{m}{m}$ & $\infty$ & $\bar{f}$ & f & \& \\
\hline 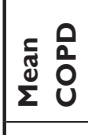 & 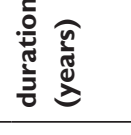 & $\sigma$ & 尽 & $\frac{\alpha}{z}$ & 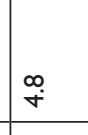 & $\frac{\alpha}{z}$ & $\stackrel{\stackrel{i}{\mathbf{I}}}{ }$ & $\frac{\mathscr{x}}{z}$ & 金 & a. & 范 & $\stackrel{\circ}{\circ}$ & $\stackrel{m}{=}$ & $\infty$ & $\infty$ \\
\hline 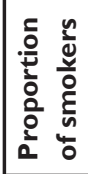 & & m & m & $\frac{\alpha}{z}$ & 8 & $\hat{\lambda}$ & m & $\frac{\tilde{z}}{z}$ & 8 & m & הి & $\bar{\gamma}$ & $\frac{\tilde{\alpha}}{z}$ & m & m \\
\hline 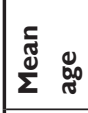 & & 6 & ڤ & \pm & $\overline{0}$ & $\stackrel{\tilde{z}}{z}$ & $\infty$ & : & త & 6 & $\overline{0}$ & 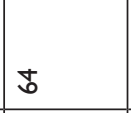 & I & ณ & อ̊ \\
\hline 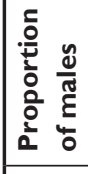 & & 8 & $\stackrel{\infty}{\infty}$ & ณ & $\stackrel{\infty}{+}$ & $\frac{\alpha}{z}$ & $\sigma$ & 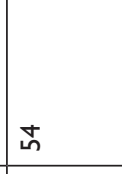 & œ & 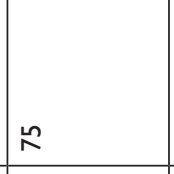 & I & $\infty$ & ڤ & 走 & $\stackrel{\infty}{\wedge}$ \\
\hline 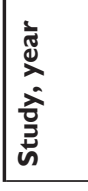 & & 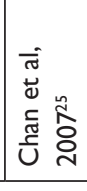 & 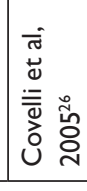 & 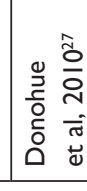 & 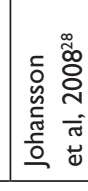 & 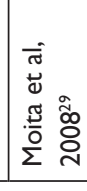 & 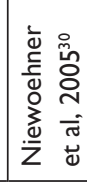 & 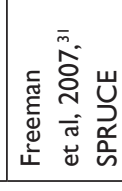 & 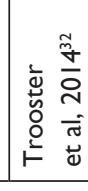 & 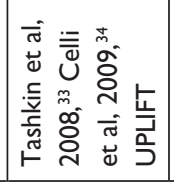 & 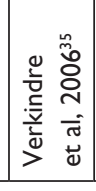 & 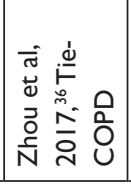 & 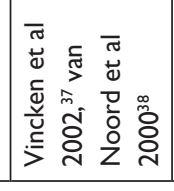 & 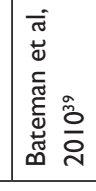 & 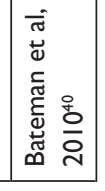 \\
\hline
\end{tabular}




\begin{tabular}{|c|c|c|c|c|c|c|c|c|c|c|c|c|c|}
\hline 3 & 3 & 3 & 3 & 3 & 30 & 3 & 吕 & 3 & 吕 & 艿 & 3 & 3 & 3 \\
\hline $\begin{array}{l}\frac{\overline{5}}{\mathrm{~b} 0} \\
\frac{\bar{I}}{\mathrm{I}}\end{array}$ & 3 & 3 & 3 & 3 & $\begin{array}{l}\frac{\bar{b}}{\mathrm{~b} 0} \\
\frac{\bar{I}}{\mathrm{I}}\end{array}$ & \begin{tabular}{|l}
$\frac{\bar{b}}{\underline{\underline{b}}}$ \\
$\underline{\underline{I}}$
\end{tabular} & 3 & 吕 & $\begin{array}{l}\frac{\bar{c}}{\underline{\underline{0}}} \\
\underline{\underline{I}}\end{array}$ & 3 & 3 & 3 & 3 \\
\hline 芆 & 3 & 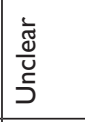 & 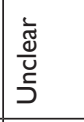 & \begin{tabular}{|l}
$\frac{\vec{b}}{\mathrm{~b}}$ \\
$\underline{\underline{\underline{I}}}$
\end{tabular} & 3 & 3 & $\begin{array}{l}\frac{i}{\tilde{\Xi}} \\
\frac{\tilde{\omega}}{\mathrm{J}} \\
\bar{J}\end{array}$ & 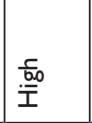 & $\begin{array}{l}\frac{i}{\tilde{d}} \\
\frac{\tilde{U}}{\mathrm{~L}} \\
\bar{J}\end{array}$ & 0 & 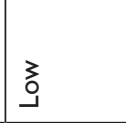 & 罣 & 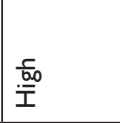 \\
\hline 3 & 光 & 3 & 3 & \begin{tabular}{|l} 
点 \\
$\underline{\underline{I}}$
\end{tabular} & 夰 & 3 & 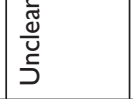 & 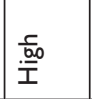 & 3 & 3 & ?o & 泉 & 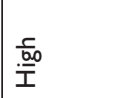 \\
\hline 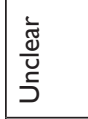 & 3 & 3 & 光 & 菏 & 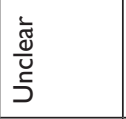 & 岁 & 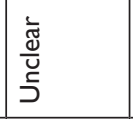 & 3 & 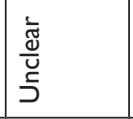 & 3 & 吕 & 3 & 3 \\
\hline 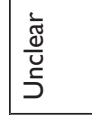 & 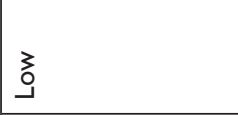 & 3 & 3 & 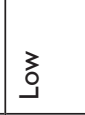 & 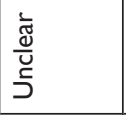 & 3 & \begin{tabular}{|l|l}
$\frac{i}{\tilde{J}}$ \\
$\frac{\tilde{J}}{\tilde{J}}$ \\
$\underline{J}$
\end{tabular} & 3 & 岁 & 㝊 & 吕 & 㝊 & 告 \\
\hline 受 & 寽 & 号 & 孚 & 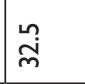 & 号 & 孚 & $\approx \sim$ & $\begin{array}{l}\stackrel{2}{2} \\
\dot{j} \\
\end{array}$ & 产 & 0 & 으 & 㐫 & 음 \\
\hline $\bar{\sigma}$ & $\stackrel{\infty}{+}$ & f & in & in & 亡े & 亡ั & กิ & นn & in & 8 & J & กิ & $\hat{m}$ \\
\hline 으 & $\stackrel{\mathscr{c}}{\mathrm{Z}}$ & 魚 & 兰 & 产 & $\stackrel{\varrho}{z}$ & 产 & 产 & フู & $\begin{array}{l}\text { tú } \\
\text { مُ }\end{array}$ & 웃 & $\stackrel{m}{r}$ & กี & $\stackrel{N}{N}$ \\
\hline 우 & $\hat{m}$ & 苟 & in & $\stackrel{\infty}{+}$ & กี & $\hat{f}$ & 兰 & 우 & นุ & $\underset{+}{\infty}$ & 우 & $\stackrel{\sim}{\sim}$ & $\stackrel{\infty}{m}$ \\
\hline ذ & ○ & ก) & ț & tे & to & జ & กิ & t & กิ & ก & น & น & జ \\
\hline ఖొ & $\overline{6}$ & ธ & $\pi$ & 6 & กิ & œ & น & $\stackrel{2}{\kappa}$ & 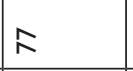 & $\pi$ & $\stackrel{2}{\circ}$ & นั & 音 \\
\hline 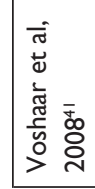 & 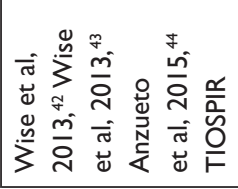 & 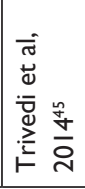 & 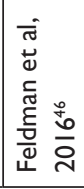 & 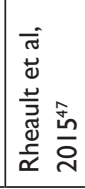 & 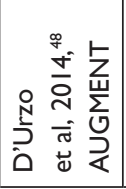 & 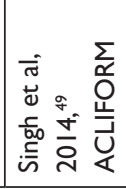 & 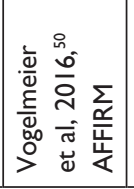 & 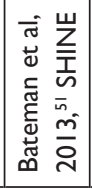 & 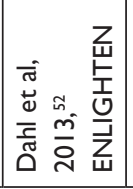 & 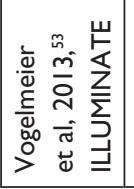 & 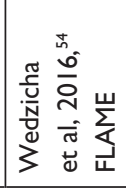 & 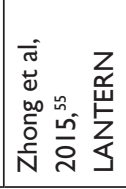 & 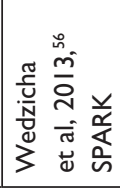 \\
\hline
\end{tabular}




\begin{tabular}{|c|c|c|c|c|c|c|c|c|c|c|c|c|c|}
\hline 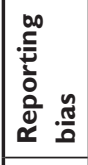 & 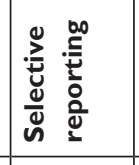 & 3 & ؛े & 3 & 吕 & 吕 & 3 & 音 & 3 & 3 & 3 & 3 & 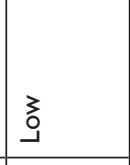 \\
\hline 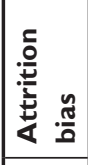 & 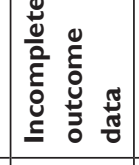 & 3 & o & 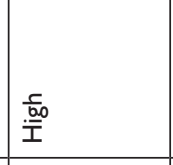 & \begin{tabular}{|l} 
豪 \\
I
\end{tabular} & 3 & 3 & 音 & 3 & 3 & 艿 & 3 & 3 \\
\hline 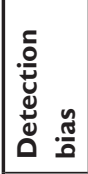 & 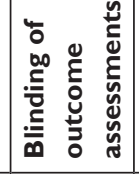 & 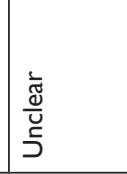 & $\begin{array}{l}\frac{\bar{d}}{\bar{d}} \\
\frac{\tilde{u}}{5}\end{array}$ & 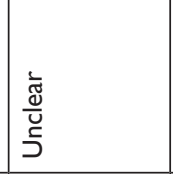 & \begin{tabular}{|l}
$\frac{1}{\tilde{J}}$ \\
$\frac{\tilde{u}}{5}$ \\
5 \\
\end{tabular} & 当 & \begin{tabular}{|l}
$\frac{1}{\tilde{J}}$ \\
$\frac{u}{5}$ \\
5 \\
\end{tabular} & 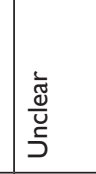 & 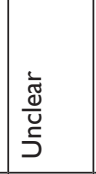 & 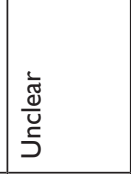 & 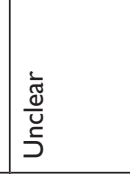 & 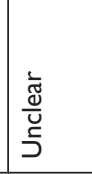 & 3 \\
\hline 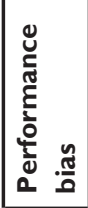 & 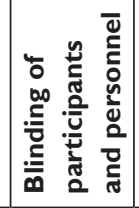 & 3 & ڤે & 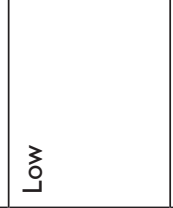 & 30 & 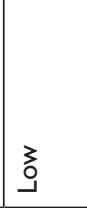 & 3 & ڤે & 3 & 3 & 3 & 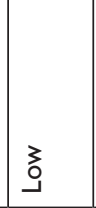 & 3 \\
\hline 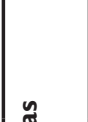 & 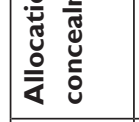 & 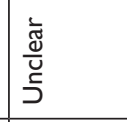 & 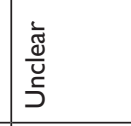 & 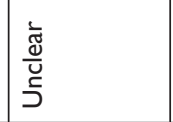 & \begin{tabular}{|l}
$\frac{5}{\tilde{m}}$ \\
$\frac{u}{5}$
\end{tabular} & 3 & 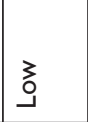 & 音 & 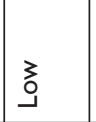 & 3 & 3 & 3 & 3 \\
\hline 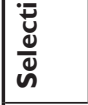 & 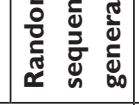 & 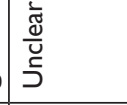 & 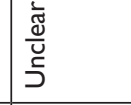 & $3_{0}^{3}$ & 吕 & 3. & 3 & $3_{0}^{3}$ & 3 & 3 & 3 & 3 & 吕 \\
\hline 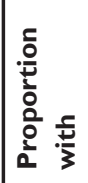 & 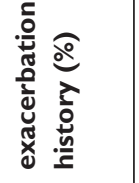 & 魚 & 魚 & 兑 & 魚 & 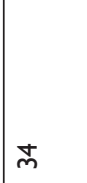 & $\frac{\alpha}{z}$ & 魚 & $\frac{\alpha}{z}$ & $\begin{array}{l}\stackrel{0}{\infty} \\
\stackrel{\infty}{q}\end{array}$ & 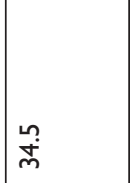 & 兑 & 㐱 \\
\hline 离离 & & 另 & 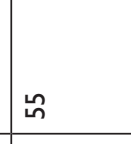 & in & in & 8 & f & f & f & क् & क & $f$ & q \\
\hline 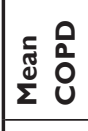 & 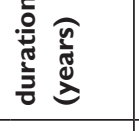 & $\frac{\alpha}{z}$ & $\frac{\tilde{x}}{\mathrm{z}}$ & $\frac{\alpha}{z}$ & $\frac{\mathscr{\alpha}}{z}$ & $\underline{\underline{\alpha}}$ & $\frac{\mathscr{\alpha}}{z}$ & 孚 & $\frac{\alpha}{z}$ & 兑 & 兑 & $\stackrel{o}{z}$ & $\frac{\alpha}{z}$ \\
\hline 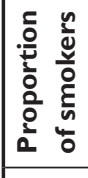 & & q & f & ñ & m & in & 古 & aे & in & f & \& & in & F \\
\hline 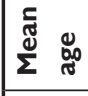 & & ڤே & : & 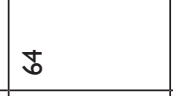 & \pm & t & 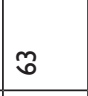 & 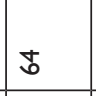 & $\ddot{0}$ & t & 古 & త & ๓ి \\
\hline 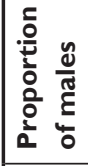 & & 8 & 0 & $\approx$ & $\approx$ & 8 & nิ & $\approx$ & $\pi$ & $\stackrel{\infty}{๖}$ & $\infty$ & $\stackrel{\infty}{\infty}$ & 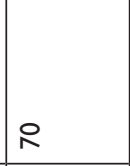 \\
\hline 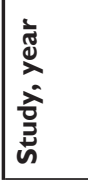 & & 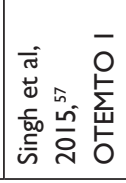 & 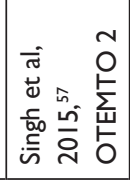 & 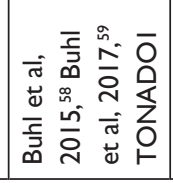 & 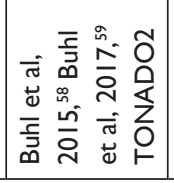 & 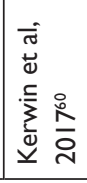 & 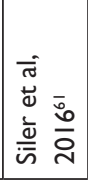 & 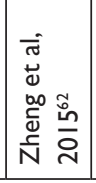 & 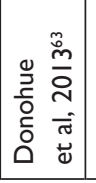 & 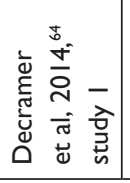 & 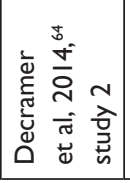 & 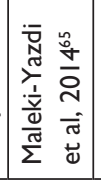 & 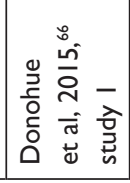 \\
\hline
\end{tabular}




\begin{tabular}{|c|c|c|c|c|c|c|c|c|c|c|c|c|c|c|c|}
\hline قُ & 3 & 3 & 3 & 苟 & 当 & 罣 & 告 & 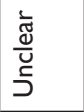 & 3. & 朵 & 告 & 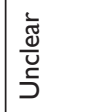 & 雚 & 艿 & 艿 \\
\hline 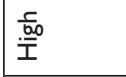 & 3 & 朵 & 3 & 3 & 3 & 3 & 3 & ơ & 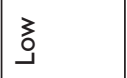 & 苞 & 3 & 3 & 3 & 苟 & 苟 \\
\hline قu & 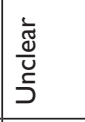 & 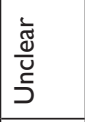 & 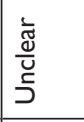 & 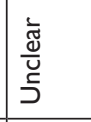 & 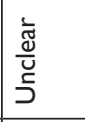 & 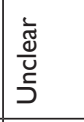 & 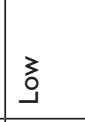 & 3 & 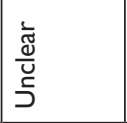 & \begin{tabular}{|l}
$\frac{1}{\bar{J}}$ \\
$\frac{\tilde{d}}{\breve{v}}$ \\
5
\end{tabular} & $\begin{array}{l}\frac{i}{\tilde{\Xi}} \\
\frac{\tilde{U}}{\breve{J}} \\
\end{array}$ & 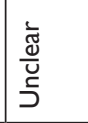 & 罣 & $3_{0}^{3}$ & 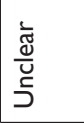 \\
\hline 3 & 3 & 3 & 3 & 3 & 3 & 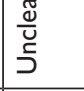 & 3 & 3 & 3 & 夰 & 3 & 3 & 3 & 3 & 3 \\
\hline 芆 & 3 & 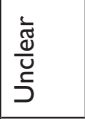 & 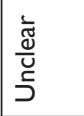 & 每 & 光 & 盛 & 岁 & 光 & 告 & 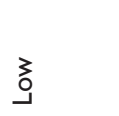 & 苟 & 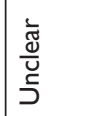 & 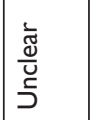 & 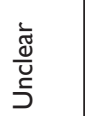 & 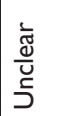 \\
\hline 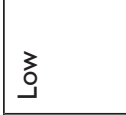 & 3 & 苟 & 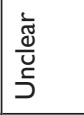 & 3 & 当 & 3 & 3 & 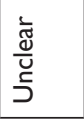 & o & 苟 & 岁 & 吕 & 罣 & 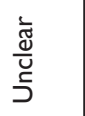 & 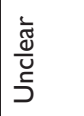 \\
\hline 寽 & 0 & 으 & 으 & 兰 & $\approx$ & ส & 岀 & 号 & 号 & 寽 & 兴 & ळ & 号 & 号 & 孚 \\
\hline 요 & in & 우 & 우 & 兰 & $\stackrel{\infty}{+}$ & $\stackrel{\infty}{+}$ & in & $\stackrel{0}{+}$ & $\stackrel{\infty}{+}$ & $g$ & $\stackrel{\infty}{\stackrel{\infty}{+}}$ & $\stackrel{\infty}{+}$ & 号 & $\mathcal{f}$ & $\mp$ \\
\hline 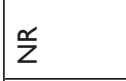 & $\stackrel{\mathscr{\alpha}}{\mathrm{z}}$ & $\stackrel{o}{z}$ & $\stackrel{ }{0}$ & 兰 & 亮 & 亮 & 兴 & 号 & 兑 & 号 & 孚 & $\stackrel{\mathscr{c}}{Z}$ & 寽 & $\frac{\mathscr{Q}}{Z}$ & 号 \\
\hline กิ & in & F & $\bar{F}$ & $\hat{m}$ & फे & กิ & กิ & $\stackrel{0}{+}$ & นn & นn & 织 & $\stackrel{\mathscr{c}}{Z}$ & in & $\stackrel{\leftrightarrow}{q}$ & 온 \\
\hline ț & $\widetilde{\sigma}$ & ஸి & ๓) & $\infty$ & $\widetilde{\sigma}$ & ธ & ๓) & i & $\overline{6}$ & $\overline{6}$ & $\overline{6}$ & ஸి & ๓) & t & 0 \\
\hline$\stackrel{\circ}{2}$ & 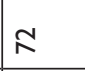 & $\infty$ & tे & $\bar{\infty}$ & $\hat{\imath}$ & $\mathbb{N}$ & 号 & $\infty$ & 。 & 。 & a & $\infty$ & ळ & to & o \\
\hline 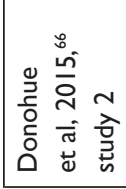 & 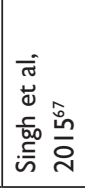 & 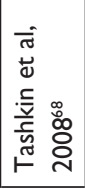 & 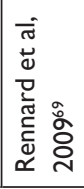 & 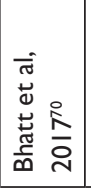 & 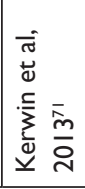 & 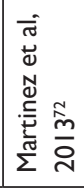 & 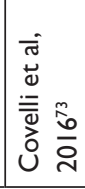 & 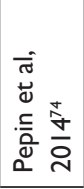 & 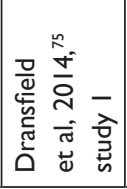 & 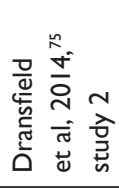 & 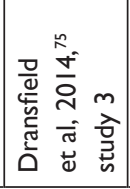 & 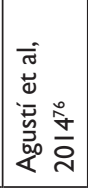 & 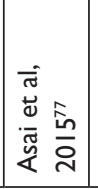 & 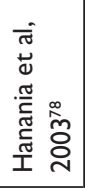 & 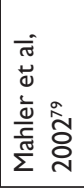 \\
\hline
\end{tabular}




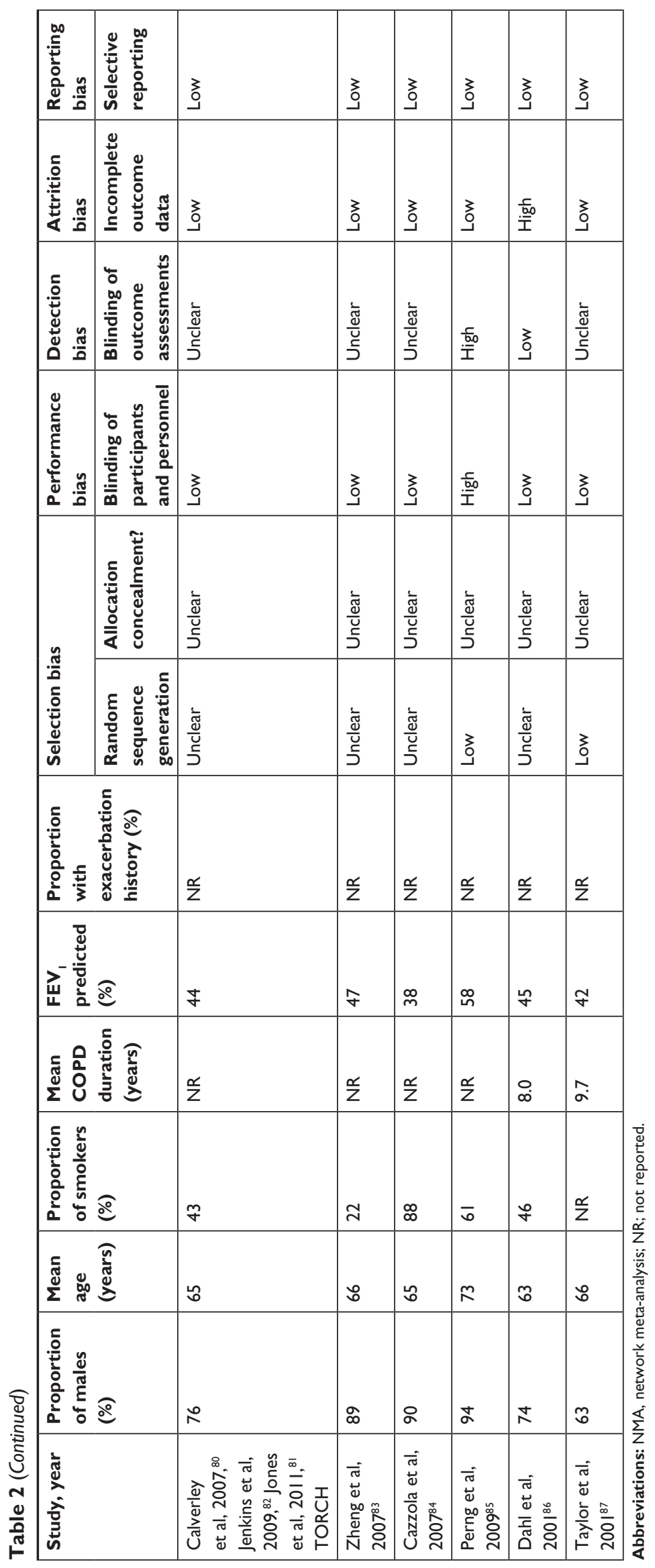




\begin{tabular}{|c|c|c|c|c|c|c|c|c|c|c|c|c|}
\hline & 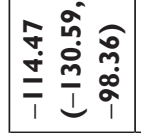 & 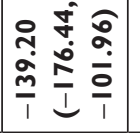 & 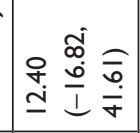 & $\overleftarrow{z}$ & 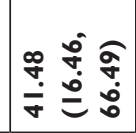 & 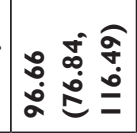 & $\S$ & 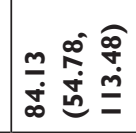 & z & 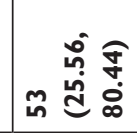 & 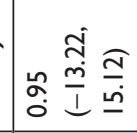 & $\S$ \\
\hline & 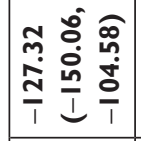 & 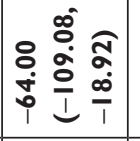 & 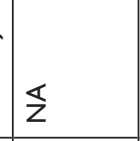 & $\S$ & $\S$ & $\S$ & 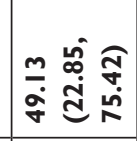 & 乏 & $\S$ & $\S$ & $\S$ & $\S$ \\
\hline & 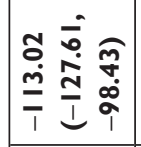 & 乏 & $\S$ & そ & そ & z & z & z & 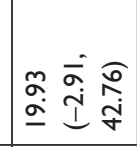 & z & 乏 & $\bar{\varepsilon}$ \\
\hline & 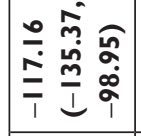 & $\S$ & 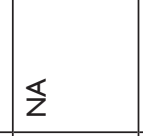 & 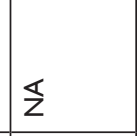 & $\S$ & $\S$ & $\S$ & 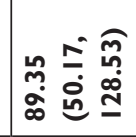 & z & m & 这 & 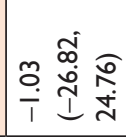 \\
\hline & 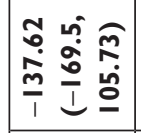 & $\S$ & $\S$ & $\S$ & z & 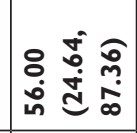 & $\S$ & $\S$ & $\S$ & 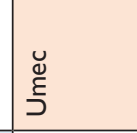 & 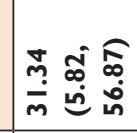 & 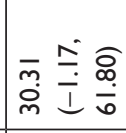 \\
\hline & 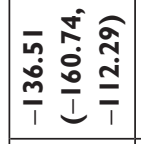 & $\S$ & $\S$ & 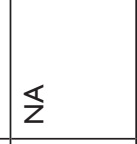 & $\S$ & $\S$ & $\S$ & $\S$ & $\begin{array}{l}\xi \\
\frac{\xi}{0} \\
\frac{0}{4} \\
\end{array}$ & 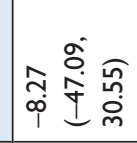 & 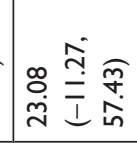 & 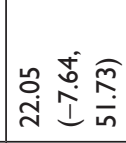 \\
\hline & 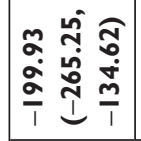 & $\S$ & $\S$ & $\S$ & 乏 & $\S$ & $\S$ & 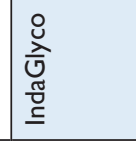 & 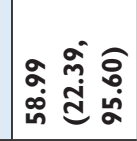 & 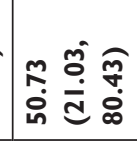 & 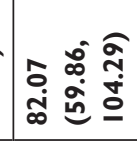 & 蓆 \\
\hline & 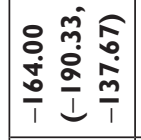 & そ & $\S$ & $\S$ & z & z & $\begin{array}{l}\stackrel{0}{0} \\
\stackrel{\circ}{\circ}\end{array}$ & 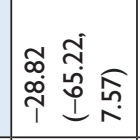 & 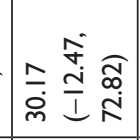 & 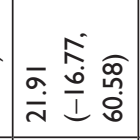 & 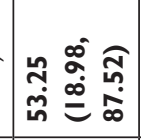 & 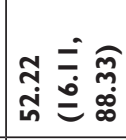 \\
\hline & 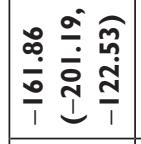 & 乏 & $\S$ & $\stackrel{\nwarrow}{z}$ & 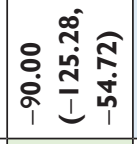 & 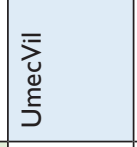 & 员触 & 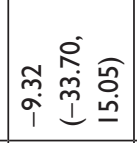 & 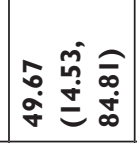 & 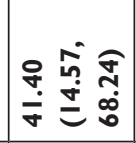 & 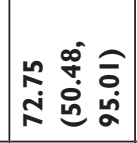 & 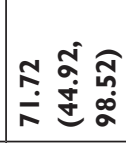 \\
\hline & 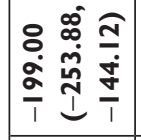 & $\S$ & 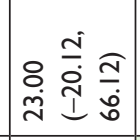 & $\S$ & 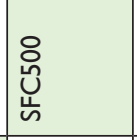 & 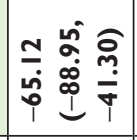 & 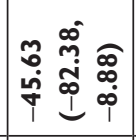 & 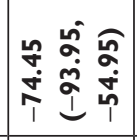 & 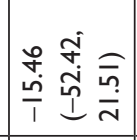 & 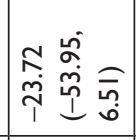 & 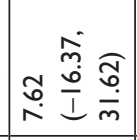 & 空 \\
\hline & 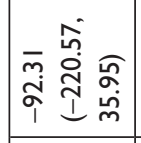 & 乏 & 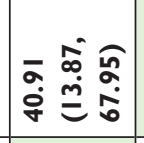 & 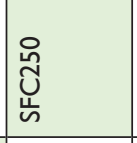 & 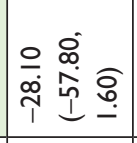 & 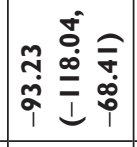 & 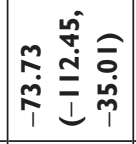 & 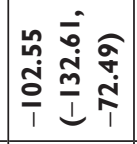 & 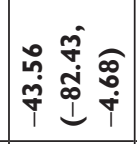 & 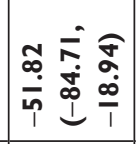 & 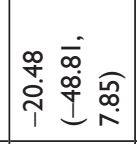 & 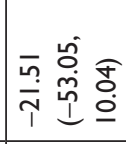 \\
\hline & 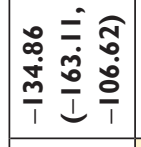 & 乏 & $\underset{\text { 立 }}{\sum}$ & 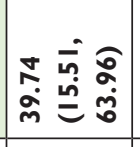 & 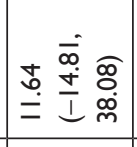 & 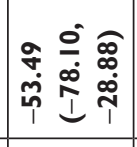 & 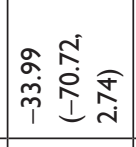 & 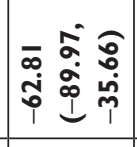 & 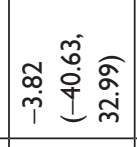 & 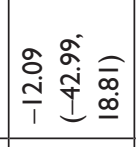 & 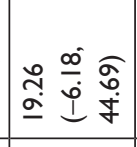 & 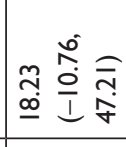 \\
\hline & 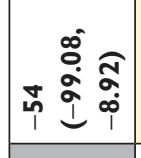 & 吾 & 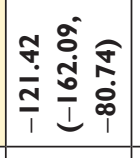 & 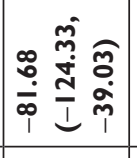 & 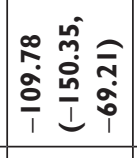 & 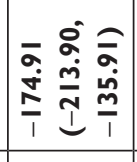 & 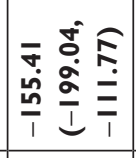 & 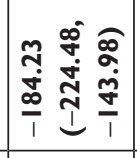 & 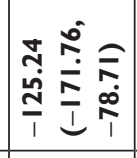 & 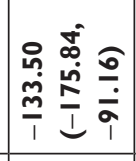 & 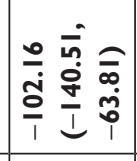 & ô \\
\hline & $\begin{array}{l}\circ \\
\stackrel{8}{0} \\
\frac{\pi}{0} \\
\frac{0}{0} \\
\end{array}$ & 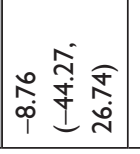 & 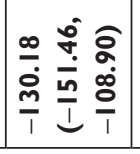 & 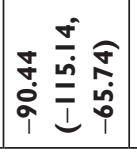 & 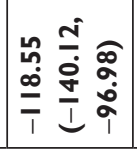 & 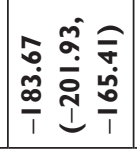 & 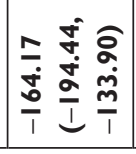 & 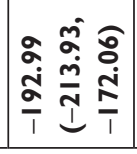 & 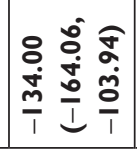 & 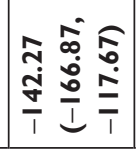 & 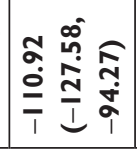 & 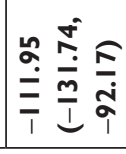 \\
\hline
\end{tabular}




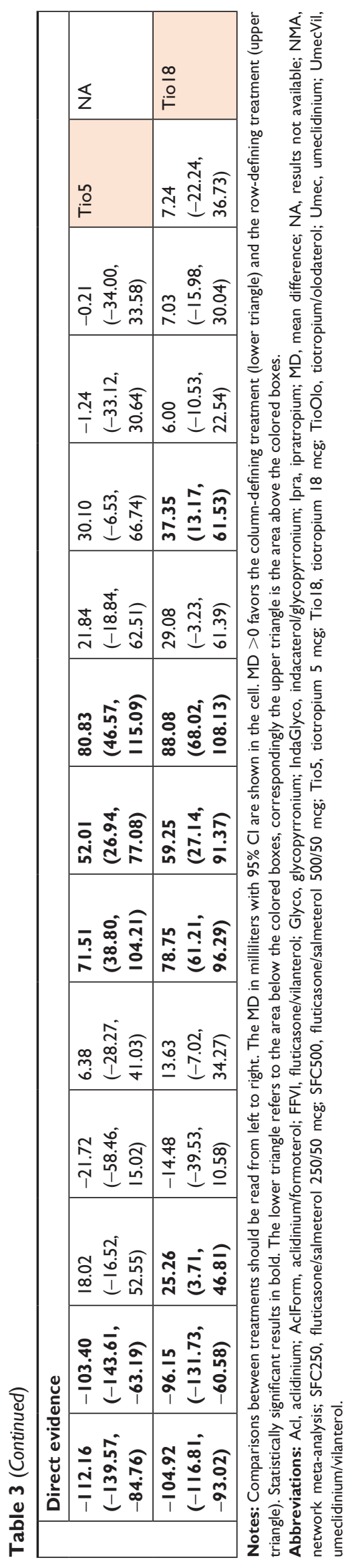

\begin{tabular}{|c|c|c|c|c|c|}
\hline & 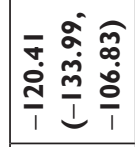 & 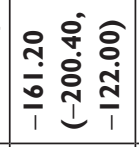 & $\mathbb{z}$ & $\S$ & $\mathbb{z}$ \\
\hline & 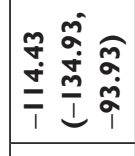 & $\S$ & 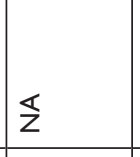 & $\S$ & $\mathbb{z}$ \\
\hline & 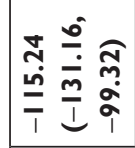 & 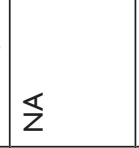 & $\Sigma$ & 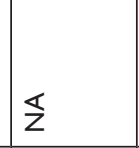 & $\mathbb{z}$ \\
\hline & 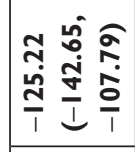 & $\underline{z}$ & $\S$ & z & $\mathbb{Z}$ \\
\hline & 葡 & $\underline{z}$ & 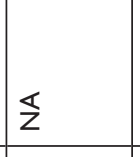 & $\mathbb{z}$ & $\mathbb{z}$ \\
\hline & 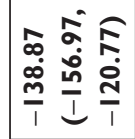 & $\mathbb{z}$ & $\S$ & $\mathbb{z}$ & $\mathbb{Z}$ \\
\hline & 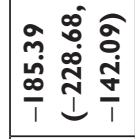 & $\underline{z}$ & $\mathbb{Z}$ & $\S$ & $\mathbf{z}$ \\
\hline & 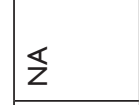 & $\frac{\pi}{z}$ & $\S$ & $\frac{\pi}{z}$ & $\mathbb{z}$ \\
\hline & 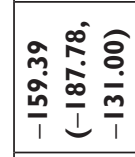 & $\mathbb{z}$ & $\mathbb{z}$ & $\S$ & 乏 \\
\hline & 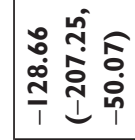 & $\mathbb{z}$ & 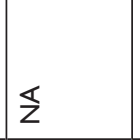 & $\mathbb{z}$ & $\mathbb{Z}$ \\
\hline & 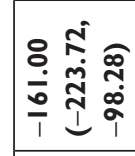 & $\frac{\pi}{z}$ & $\mathbb{z}$ & 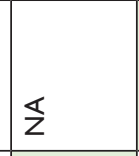 & 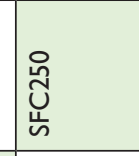 \\
\hline & 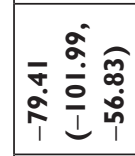 & $\mathbb{z}$ & ż & 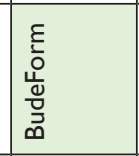 & 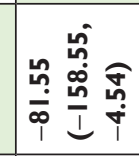 \\
\hline & 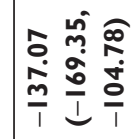 & $\frac{1}{z}$ & $\underset{\Psi}{\bar{H}}$ & 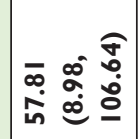 & 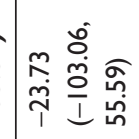 \\
\hline & $\underline{z}$ & 吾 & 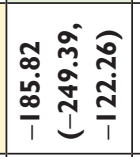 & 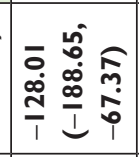 & 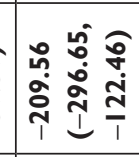 \\
\hline 苞 & 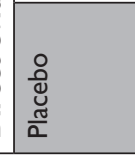 & 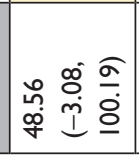 & 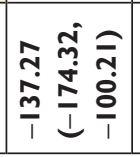 & 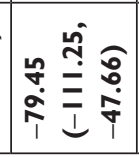 & 舁 \\
\hline
\end{tabular}




\begin{tabular}{|c|c|c|c|c|c|c|c|c|c|}
\hline$\underline{z}$ & 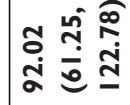 & $\frac{s}{z}$ & 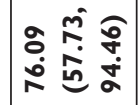 & $\underline{z}$ & $\underline{z}$ & 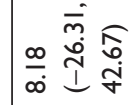 & $\underline{z}$ & $\frac{\mathbb{z}}{z}$ & 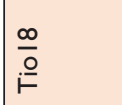 \\
\hline$\underline{z}$ & $\mathbb{z}$ & 雚 & 乏 & $\underline{z}$ & $\underline{z}$ & $\frac{s}{z}$ & $\underline{z}$ & 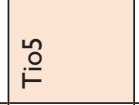 & 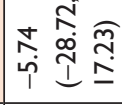 \\
\hline$\underline{z}$ & $\frac{\pi}{z}$ & $\S$ & $\S$ & 产 & $\underline{z}$ & $\S$ & $\bar{\Psi}$ & 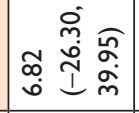 & 足 \\
\hline$\underline{z}$ & $\frac{\mathbb{s}}{z}$ & $\frac{s}{z}$ & 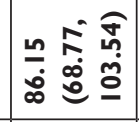 & $\frac{\pi}{z}$ & $\frac{s}{z}$ & 总 & 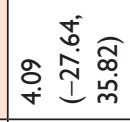 & 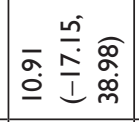 & 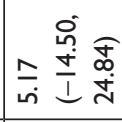 \\
\hline$\underline{z}$ & 定 & $\frac{\pi}{z}$ & $\frac{\mathbb{z}}{z}$ & $\underline{z}$ & 造 & 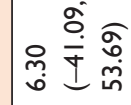 & 㡍高 & 空豙递 & f. \\
\hline$\underline{z}$ & $\frac{\mathbb{z}}{z}$ & $\underline{z}$ & $\S$ & \begin{tabular}{|l}
$\frac{E}{0}$ \\
$\frac{0}{4}$ \\
$\frac{4}{4}$
\end{tabular} & 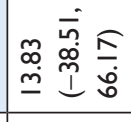 & 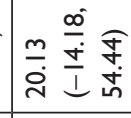 & 弪高总 & 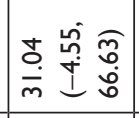 & 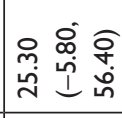 \\
\hline 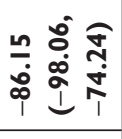 & $\frac{\pi}{z}$ & $\underline{z}$ & 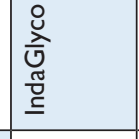 & 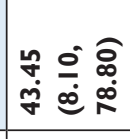 & 帘 & 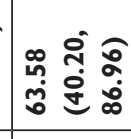 & 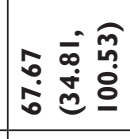 & 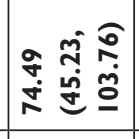 & 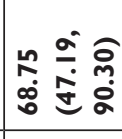 \\
\hline$\underline{\Sigma}$ & $\S$ & \begin{tabular}{l}
$\stackrel{\circ}{0}$ \\
$\stackrel{0}{\circ}$ \\
\hdashline
\end{tabular} & 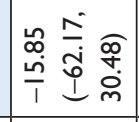 & 总 & 竞 & 色 & 它 & 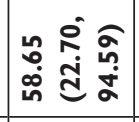 & 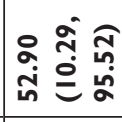 \\
\hline$\underline{z}$ & 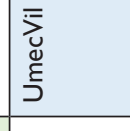 & 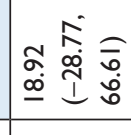 & 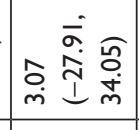 & 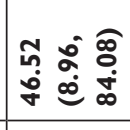 & 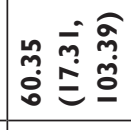 & 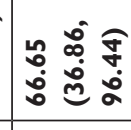 & 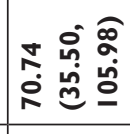 & 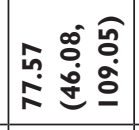 & 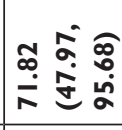 \\
\hline 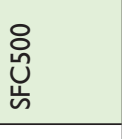 & 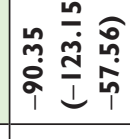 & 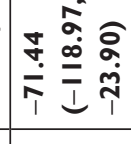 & 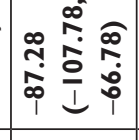 & 辛 & 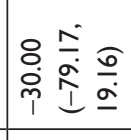 & 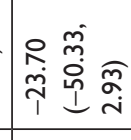 & 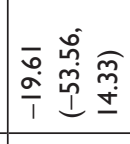 & 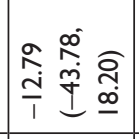 & 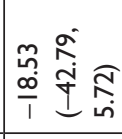 \\
\hline 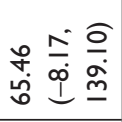 & 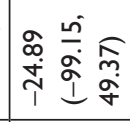 & مُ & 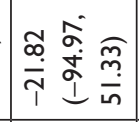 & 言离命 & 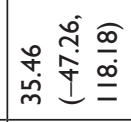 & 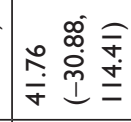 & 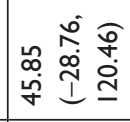 & 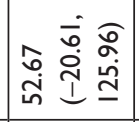 & 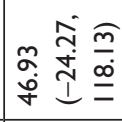 \\
\hline $\begin{array}{l}\infty \\
0 \\
0 \\
0 \\
\end{array}$ & 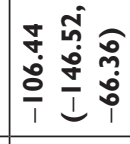 & 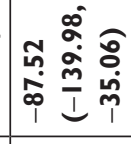 & 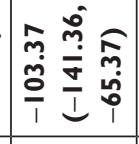 & 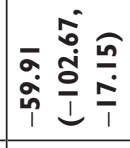 & 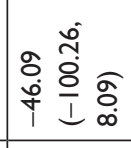 & 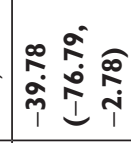 & 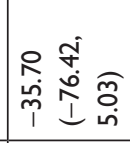 & 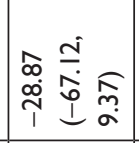 & 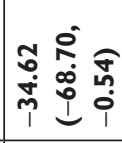 \\
\hline 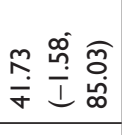 & 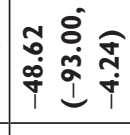 & 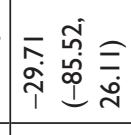 & 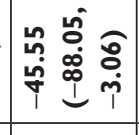 & 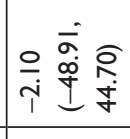 & 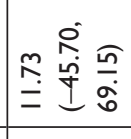 & 宓 & ف. & 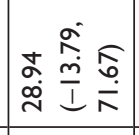 & 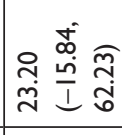 \\
\hline 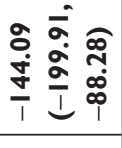 & 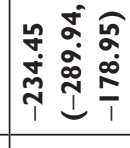 & 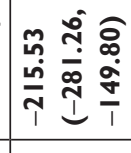 & 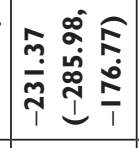 & 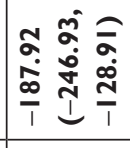 & 资 & 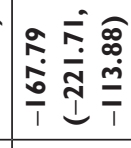 & 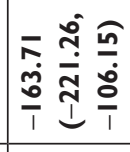 & 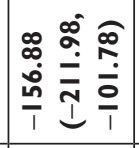 & 柋高 \\
\hline 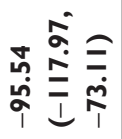 & 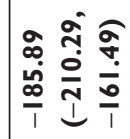 & 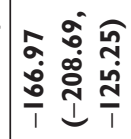 & 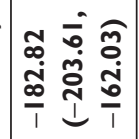 & 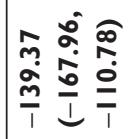 & 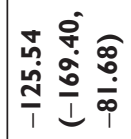 & 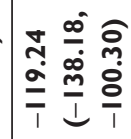 & 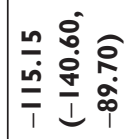 & 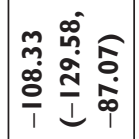 & 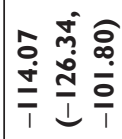 \\
\hline
\end{tabular}

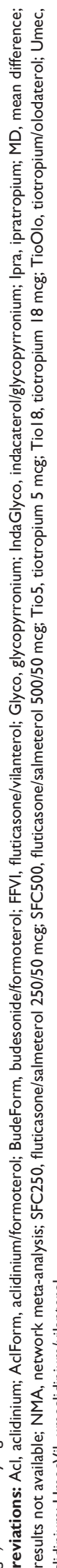


to $-1 \mathrm{~mL}$ favoring Tio18). When compared to the LAMA/ LABAs, ICS/LABAs conferred significantly less improvement at weeks 12 and 24. The MDs in improvements in $\mathrm{FEV}_{1}$ between the ICS/LABAs and LAMA/LABAs were smaller with AclForm vs ICS/LABAs than with the other three LAMA/LABAs (IndaGlyco, TioOlo and UmecVil).

\section{Transition dyspnea index}

TDI scores were reported in 28 studies at week 12 and in 19 studies at week 24. Significantly greater improvements in TDI scores were observed with all LAMAs, LAMA/LABAs and ICS/LABAs compared to SAMA and placebo at weeks 12 and 24 (Tables S2 and $\underline{\text { S3 }}$ ). There were no significant differences between the LAMA/LABAs except UmecVil vs TioOlo at week 12 (MD $-0.51,95 \%$ CI -0.94 to -0.07 , favoring TioOlo). The difference was not considered to be clinically significant (MCID $\geq 1$ point increase). There were no significant differences in TDI scores between all LAMA-LAMA comparisons at weeks 12 and 24. There were, however, some statistically significant improvements in TDI scores for LAMA/LABAs compared to LAMAs. Nonetheless, the magnitudes of difference in TDI scores were all lower than the MCID of $\geq 1$ unit for these comparisons. Similarly, there were some statistically significant (but not clinically significant) differences when LAMA/LABAs were compared to ICS/LABAs. There were no significant differences in TDI scores when LAMAs were compared to ICS/ LABAs at both time points.

In terms of the proportion of TDI score responders (who achieved a minimum of 1-point improvement in TDI score), results were reported in 15 studies at week 12 and in 16 studies at week 24. Study participants treated with LAMAs, LAMA/LABAs or ICS/LABAs were more likely to achieve improvement in TDI score than those receiving placebo or SAMA (Tables S4 and S5). There was some evidence to show that LAMA/LABA treatment had higher odds of achieving TDI score improvement compared to LAMAs (UmecVil vs Tio1 8 at week 12: OR 1.49, 95\% CI 1.16-1.93; IndaGlyco vs Tio18 at week 24: OR 1.45, 95\% CI 1.11-1.89; AclForm vs Tio18 at week 24: OR 1.37, 95\% CI 1.04-1.80). There were no significant differences in TDI score improvement when ICS/LABAs were compared with LAMAs or LAMA/ LABAs.

\section{St George's Respiratory Questionnaire}

Health-related quality-of-life (HRQoL) benefits as measured by SGRQ scores were reported in 34 studies at week 12 and in 29 studies at week 24. All LAMAs, LAMA/LABAs and
ICS/LABAs showed statistically significant improvement in SGRQ score compared to placebo at week 12; however, the point estimates did not achieve clinical significance (MCID $\geq 4$-point decrease) for SFC250, Acl, Glyco, Tio5 and Tio18 (Table S6). At week 24, only some of the LAMAs and LAMA/LABAs showed statistically significant improvements in HRQoL vs placebo (Table S7), but none of the results reached clinical significance. A similar trend was seen when LAMAs, LAMA/LABAs and ICS/LABAs were compared to SAMA. Within each class, the LAMAs, LAMA/ LABAs and ICS/LABAs led to similar HRQoL improvements at weeks 12 and 24. While there were some statistically significant differences between LAMA/LABAs and LAMAs, these differences were not clinically significant.

In terms of the proportion of SGRQ score responders (achieving at least a 4-point reduction in SGRQ), results were reported in 21 studies at week 12 and in 19 studies at week 24. At both time points, all LAMAs, LAMA/LABAs and ICS/LABAs led to a significantly higher proportion of study participants achieving an improvement in SGRQ score compared to placebo (Tables $\mathrm{S} 8$ and $\underline{\mathrm{S} 9}$ ). Relative to SAMA, only IndaGlyco and TioOlo showed a statistically significant difference in SGRQ responders at week 12 (Ipra vs IndaGlyco: OR 0.61, 95\% CI 0.40-0.94; Ipra vs TioOlo: OR $0.53,95 \%$ CI 0.33-0.85). There were no significant differences within the LAMA, LAMA/LABA and ICS/LABA classes at weeks 12 and 24 for SGRQ responders; however, some evidence was available to suggest that the LAMA/ LABAs led to a higher proportion of SGRQ responder compared to the LAMAs at both weeks 12 and 24.

\section{Adverse events}

Incidences of AEs were reported in 17 studies at week 12 and in 27 studies at week 24. There were no significant differences in the proportion of patients who experienced AEs for any comparison at week 12 (Table S10). At week 24, a significantly higher proportion of patients receiving SFC500 had AEs compared to those receiving IndaGlyco (SFC500 vs IndaGlyco: OR 1.34, 95\% CI 1.04-1.72; Table S11). This translated to a Number Needed to Harm of 14. No other significant differences with respect to AEs were found in other comparisons.

\section{Ranking of treatments}

In general, the LAMA/LABAs ranked the highest among the different drug classes for lung function improvement $\left(\mathrm{FEV}_{1}\right)$ at weeks 12 and 24, while placebo and SAMA ranked the lowest. The SUCRA values for LAMA/LABAs 
Table 5 SUCRA values for all interventions for each outcome

\begin{tabular}{|c|c|c|c|c|c|c|c|c|c|c|c|c|}
\hline & \multicolumn{2}{|l|}{ FEV $_{1}$} & \multicolumn{2}{|c|}{ TDI score } & \multicolumn{2}{|c|}{ TDI responder } & \multicolumn{2}{|c|}{ SGRQ score } & \multicolumn{2}{|c|}{ SGRQ responder } & \multicolumn{2}{|c|}{ Adverse events } \\
\hline & $\begin{array}{l}12 \\
\text { weeks }\end{array}$ & $\begin{array}{l}24 \\
\text { weeks }\end{array}$ & $\begin{array}{l}12 \\
\text { weeks }\end{array}$ & \begin{tabular}{|l}
24 \\
weeks
\end{tabular} & $\begin{array}{l}12 \\
\text { weeks }\end{array}$ & $\begin{array}{l}24 \\
\text { weeks }\end{array}$ & $\begin{array}{l}12 \\
\text { weeks }\end{array}$ & \begin{tabular}{|l}
24 \\
weeks
\end{tabular} & $\begin{array}{l}12 \\
\text { weeks }\end{array}$ & $\begin{array}{l}24 \\
\text { weeks }\end{array}$ & $\begin{array}{l}12 \\
\text { weeks }\end{array}$ & $\begin{array}{l}24 \\
\text { weeks }\end{array}$ \\
\hline Placebo & 2.6 & 6.9 & 2.4 & 9.6 & 1.8 & 10.8 & 0.7 & 6.8 & 1.0 & 2.0 & 25.2 & 62.1 \\
\hline Tiol 8 & 29.5 & 42.6 & 29.7 & 40.1 & 39.1 & 38.5 & 32.9 & 42.1 & 33.4 & 30.2 & 58.9 & 62.6 \\
\hline Tio5 & 40.8 & 36.1 & 59.5 & NA & NA & NA & 27.4 & 44.6 & NA & 35.2 & 75.4 & NA \\
\hline $\mathrm{Acl}$ & 39.6 & 43.6 & 56 & 47.6 & 36.6 & 55.4 & 23.9 & 51.7 & 20.1 & 72.7 & 63.3 & NA \\
\hline Glyco & 39.2 & 49.2 & 36.1 & 54.5 & 58.3 & 61.3 & 50.5 & 61.3 & 48.0 & 32.7 & 49.9 & 72.3 \\
\hline Umec & 72.6 & 53.9 & 42.2 & 43.8 & 58.6 & 34.2 & 68.1 & 68.1 & 38.5 & 38.1 & 31.5 & 29.4 \\
\hline AclForm & 64.5 & 66.8 & NA & 88.4 & 72.8 & 88.7 & NA & 61.6 & 68.2 & 76.9 & NA & 74.8 \\
\hline IndaGlyco & 97.6 & 91.7 & 91.6 & 94.2 & 49.1 & 93.5 & 93.1 & 60.6 & 81.7 & 76.1 & NA & 83.9 \\
\hline TioOlo & 84.2 & 82.8 & 97.8 & NA & $\mathrm{NA}$ & NA & 77.0 & 66.1 & 93.4 & 85.0 & 75.8 & NA \\
\hline UmecVil & 93.0 & 93.3 & 71.9 & 53.5 & 85.1 & 54.8 & 65.6 & 69.5 & 62.6 & 60.7 & 56.6 & 33.7 \\
\hline SFC500 & 48.9 & 24.5 & 69.9 & 66.8 & 85.6 & 62.2 & 71.1 & 19.2 & 64.6 & 72.6 & 49.3 & 33.3 \\
\hline SFC250 & 19.4 & 76.9 & 35.7 & NA & NA & NA & 53.0 & NA & NA & NA & 40.0 & 26.2 \\
\hline BudeForm & NA & 17.5 & NA & NA & NA & NA & NA & 60.5 & NA & 48.4 & NA & 23.4 \\
\hline FFVI & 63.0 & 64.0 & NA & NA & NA & NA & 75.6 & 71.9 & 70.7 & NA & 28.8 & 48.5 \\
\hline Ipra & 5.1 & 0.2 & 7.2 & 1.5 & 13.0 & 0.4 & 11.2 & 15.8 & 17.8 & 19.6 & 45.3 & NA \\
\hline
\end{tabular}

Note: The probabilities of each treatment being ranked best are represented by their SUCRA values.

Abbreviations: Acl, aclidinium; AclForm, aclidinium/formoterol; BudeForm, budesonide/formoterol; FFVI, fluticasone/vilanterol; Glyco, glycopyrronium; IndaGlyco, indacaterol/glycopyrronium; Ipra, ipratropium; NA, results not available; SFC250, fluticasone/salmeterol 250/50 mcg; SFC500, fluticasone/salmeterol 500/50 mcg; SGRQ, St George's Respiratory Questionnaire; SUCRA, Surface Under the Cumulative Ranking; TDI, transitional dyspnea index; Tio5, tiotropium 5 mcg; Tio I8, tiotropium I8 mcg; TioOlo, tiotropium/olodaterol; Umec, umeclidinium; UmecVil, umeclidinium/vilanterol.

ranged from $64.5 \%$ to $97.6 \%$ (Table 5). The trend remained constant for all outcomes, with LAMA/LABAs having the highest SUCRA scores.

\section{Discussion}

Our network meta-analysis is the first to utilize a frequentist framework to comprehensively compare the effectiveness of SAMAs, LAMAs, LAMA/LABAs and ICS/LABAs using published randomized controlled studies. A frequentist framework allowed us to make statistical inference/comparisons based on significance testing using $P$-values. With regards to lung function, our results showed that LAMAs, LAMA/ LABAs and ICS/LABAs led to a greater improvement in trough $\mathrm{FEV}_{1}$ compared with placebo and SAMA monotherapy. All LAMA/LABAs except aclidinium/formoterol were significantly better than LAMA monotherapy in improving lung function. Limited evidence also suggested LAMA/ LABAs led to greater improvements than ICS/LABAs. Of note, there was markedly more evidence available for lung function than other patient-relevant outcomes. Similar trends were, nonetheless, observed with respect to improvements in TDI and SGRQ scores, although not all results were statistically significant. Improvements with $\mathrm{FEV}_{1}$ have been correlated with improvements in quality of life as demonstrated in previous analyses. ${ }^{12}$ A recent study by Sion et al (2017) reported similar findings that LAMA/LABAs combinations were better than Tio alone or placebo. ${ }^{88}$

Our results did not show any clinically significant differences among the different LAMAs and LAMA/LABAs within their classes, for all outcomes. These results were congruent with other published network meta-analyses which compared outcomes within the drug classes. Cope et al, ${ }^{89}$ Karabis et a ${ }^{90}$ and Ismaila et a ${ }^{91}$ evaluated the comparative efficacy among the LAMA agents through a Bayesian framework and found no differences among them. Similarly, Schlueter et $\mathrm{al}^{92}$ and Huisman et $\mathrm{al}^{93}$ evaluated the comparative efficacy among LAMA/LABAs using the Bayesian approach and found no differences among all agents. Our analysis, which employs a frequentist framework and uses a network with more comprehensive treatment options (SAMA, LAMA, LAMA/LABA and ICS/LABA) for stable COPD, adds further confidence to these findings and expands the existing evidence base.

In considering the results, we need to be mindful of the limitations of the analysis. $\mathrm{FEV}_{1}$ is the only outcome that is consistently reported across the trials. Given TDI and SGRQ outcomes can be reported as either total score or proportion of responders, this resulted in many studies not reporting both types of outcome. Therefore, there was uncertainty in our analysis of TDI and SGRQ outcomes, with the results reflected in wide CIs. 
In addition, some included studies were open label (Bateman et $\mathrm{al}^{51}$ and Kerwin et $\mathrm{al}^{13}$ ), and hence were associated with a high risk of bias in terms of lack of blinding. Incomplete outcome data in some studies also may have increased uncertainty around some results. Small study bias was considered unlikely, given that most included trials had a sample size of at least 100 patients and each arm of all included comparisons had at least 50 patients. Most of the included studies were of a short duration with only 16 studies, out of the 74 , reporting outcomes beyond the 24 -week time point. Therefore, only the 12- and 24-week time points were selected for evaluation.

When performing network meta-analysis, patients from all pair-wise meta-analysis have not been randomized to different trials and randomization would, therefore, not hold across the set of trials used for the analysis. Thus, it is important to assess imbalance in patient characteristics and effect modifiers across trials to determine the face validity of the analysis. To ensure the assumptions of homogeneity and transitivity are met, the distribution of potential effect modifiers, such as gender distribution, mean age, and proportion of smokers, was assessed and found to be similar across the direct comparisons in the network. However, other effect modifiers, including mean COPD duration and proportion with exacerbation history, were not reported in the majority of the trials, which limited our ability to determine if our assumptions were met with respect to these characteristics. However, despite this limitation, it is unlikely that our results would be substantially biased given the consistency of results demonstrated between the network and direct comparison meta-analyses.

To address the potential influence of certain treatment effect modifiers, network meta-regression would have been appropriate to explore the impact of covariates on all of the data and allow for the simultaneous consideration of continuous and categorical covariates. However, we did not perform a meta-regression mainly due to the fact that the variation in $\mathrm{FEV}_{1}$ was too small, and also due to the limited number of studies available in parts of the network. Model diagnostics and adequacy are difficult to assess. Even if the network meta-regression was performed, individual patient data would be necessary to avoid ecological bias and to gain greater statistical power to detect differences in treatment effects between the effect modifiers.

It is worth noting that although we have reported the ranking of all treatments using SUCRA curves, large differences in ranking probabilities between two treatments do not necessarily mean significant difference in relative treatment effect. To achieve a more objective assessment, the magnitude of absolute benefit should be accompanied with ranking information in order to minimize potential biased interpretation.
Finally, our approach was based on individual treatments (eg, Acl, Glyco, Tio18, Tio5, Umec) instead of drug classes (eg, LAMAs) as it facilitated comparisons both within and across classes. However, this led to multiple comparisons involving all treatments from each class and, thus, difficulty in drawing conclusions at a therapeutic class level. The number of studies available for each individual treatment was also small, which may have resulted in low statistical power.

\section{Conclusion}

LAMA/LABA showed greatest improvement in lung function at weeks 12 and 24 compared with the other inhaled drug classes, while SAMA showed the least improvement. There were no significant differences among the LAMAs and LAMA/ LABAs within their respective classes. Results from our analysis may play a role in assisting clinicians make evidence-based treatment decisions and also in advising policymakers on the most effective treatments when making subsidy decisions. Other factors, including cost-effectiveness and patient preferences, may also be taken into account when determining the most optimal treatments for patients with stable COPD.

\section{Acknowledgment}

David Bin-Chia Wu left the Agency for Care Effectiveness, Ministry of Health, Singapore during the course of manuscript development.

\section{Disclosure}

The authors report no conflicts of interest in this work.

\section{References}

1. World Health Organization. The top 10 causes of death [updated January 2017]. Available from: http://www.who.int/gho/mortality_burden_disease/causes_death/top_10/en/. Accessed 17 September, 2018.

2. Global Initiative for Chronic Obstructive Lung Disease. Global strategy for the diagnosis, management and prevention of COPD. 2018. Available from: http://goldcopd.org. Accessed 17 September, 2018.

3. Rohatgi A. WebPlotDigitizer Version 4.1 [updated 8 January 2018]. Available from: https://automeris.io/WebPlotDigitizer/. Accessed 17 September, 2018.

4. Higgins JP, Altman DG, Gotzsche PC, Juni P, Moher D, Oxman AD. The Cochrane Collaboration's tool for assessing risk of bias in randomised trials. BMJ. 2011;343:d5928.

5. White IR. Multivariate random-effects meta-regression: updates to mvmeta. Stata J. 2011;11:255-270.

6. Harris RJ, Bradburn MJ, Deeks JJ, Harbord RM, Altman DG, Sterne JA. Metan: fixed and random-effects meta-analysis. Stata J. 2008;8(1):3-28.

7. Cazzola M, MacNee W, Fau MartinezFJ, et al. Outcomes for COPD pharmacological trials: from lung function to biomarkers. 2008;31(2):416-469.

8. Donohue JF. Minimal clinically important differences in COPD lung function. COPD. 2005;2(1):111-124.

9. Mahler DA, Witek TJ. The MCID of the transition dyspnea index is a total score of one unit. COPD. 2005;2(1):99-103.

10. Alma H, de Jong C, Jelusic D, et al. Health status instruments for patients with COPD in pulmonary rehabilitation: defining a minimal clinically important difference. NPJ Prim Care Respir Med. 2016;26:16041. 
11. Chaimani A, Higgins JP, Mavridis D, Spyridonos P, Salanti G. Graphical tools for network meta-analysis in STATA. PLoS One. 2013;8(10):e76654.

12. Westwood M, Bourbeau J, Jones PW, Cerulli A, Capkun-Niggli G, Worthy G. Relationship between FEV1 change and patient-reported outcomes in randomised trials of inhaled bronchodilators for stable COPD: a systematic review. Respir Res. 2011;12:40.

13. Kerwin EM, D’Urzo AD, Gelb AF, Lakkis H, Garcia Gil E, Caracta CF; ACCORD I study investigators. Efficacy and safety of a 12-week treatment with twice-daily aclidinium bromide in COPD patients (ACCORD COPD I). COPD. 2012;9(2):90-101.

14. Rennard SI, Scanlon PD, Ferguson GT, et al. ACCORD COPD II: a randomized clinical trial to evaluate the 12 -week efficacy and safety of twice-daily aclidinium bromide in chronic obstructive pulmonary disease patients. Clin Drug Investig. 2013;33(12):893-904.

15. Jones PW, Singh D, Bateman ED, et al. Efficacy and safety of twicedaily aclidinium bromide in COPD patients: the ATTAIN study. Eur Respir J. 2012;40(4):830-836.

16. Lee $\mathrm{SH}$, Lee J, Yoo KH, et al. Efficacy and safety of aclidinium bromide in patients with COPD: a Phase 3 randomized clinical trial in a Korean population. Respirology. 2015;20(8):1222-1228.

17. D'Urzo A, Ferguson GT, van Noord JA, et al. Efficacy and safety of once-daily NVA237 in patients with moderate-to-severe COPD: the GLOW1 trial. Respir Res. 2011;12:156.

18. Wang C, Sun T, Huang Y, et al. Efficacy and safety of once-daily glycopyrronium in predominantly Chinese patients with moderate-tosevere chronic obstructive pulmonary disease: the GLOW7 study. Int J Chron Obstruct Pulmon Dis. 2015;10:57-68.

19. Chapman KR, Beeh KM, Beier J, et al. A blinded evaluation of the efficacy and safety of glycopyrronium, a once-daily long-acting muscarinic antagonist, versus tiotropium, in patients with COPD: the GLOW5 study. BMC Pulm Med. 2014;14(4):4.

20. Kerwin E, Hébert J, Gallagher N, et al. Efficacy and safety of NVA237 versus placebo and tiotropium in patients with COPD: the GLOW2 study. Eur Respir J. 2012;40(5):1106-1114.

21. Ambrosino N, Foglio K, Balzano G, Paggiaro PL, Lessi P, Kesten S. Tiotropium and exercise training in COPD patients: effects on dyspnea and exercise tolerance. Int J Chron Obstruct Pulmon Dis. 2008;3(4):771-780.

22. Brusasco V, Hodder R, Miravitlles M, Korducki L, Towse L, Kesten S. Health outcomes following treatment for six months with once daily tiotropium compared with twice daily salmeterol in patients with COPD. Thorax. 2003;58(5):399-404.

23. Casaburi R, Briggs DD, Donohue JF, Serby CW, Menjoge SS, Witek TJ. The spirometric efficacy of once-daily dosing with tiotropium in stable COPD: a 13-week multicenter trial. The US Tiotropium Study Group. Chest. 2000;118(5):1294-1302.

24. Casaburi R, Mahler DA, Jones PW, et al. A long-term evaluation of once-daily inhaled tiotropium in chronic obstructive pulmonary disease. Eur Respir J. 2002;19(2):217-224.

25. Chan CK, Maltais F, Sigouin C, Haddon JM; Ford GTSAFE Study Group. A randomized controlled trial to assess the efficacy of tiotropium in Canadian patients with chronic obstructive pulmonary disease. Can Respir J. 2007;14(8):465-472.

26. Covelli H, Bhattacharya S, Cassino C, Conoscenti C, Kesten S. Absence of electrocardiographic findings and improved function with once-daily tiotropium in patients with chronic obstructive pulmonary disease. Pharmacotherapy. 2005;25(12):1708-1718.

27. Donohue JF, Fogarty C, Lotvall J, et al. Once-daily bronchodilators for chronic obstructive pulmonary disease: indacaterol versus tiotropium. Am J Respir Crit Care Med. 2010;182(2):155-162.

28. Johansson G, Lindberg A, Romberg K, Nordström L, Gerken F, Roquet A. Bronchodilator efficacy of tiotropium in patients with mild to moderate COPD. Prim Care Respir J. 2008;17(3):169-175.

29. Moita J, Bárbara C, Cardoso J, et al. Tiotropium improves FEV1 in patients with COPD irrespective of smoking status. Pulm Pharmacol Ther. 2008;21(1):146-151.
30. Niewoehner DE, Rice K, Cote C, et al. Prevention of exacerbations of chronic obstructive pulmonary disease with tiotropium, a once-daily inhaled anticholinergic bronchodilator: a randomized trial. Ann Intern Med. 2005;143(5):317-326.

31. Freeman D, Lee A, Price D. Efficacy and safety of tiotropium in COPD patients in primary care - the SPiRiva Usual CarE (SPRUCE) study. Respir Res. 2007;8:45.

32. Troosters T, Sciurba FC, Decramer M, et al. Tiotropium in patients with moderate COPD naive to maintenance therapy: a randomised placebo-controlled trial. NPJ Prim Care Respir Med. 2014;24:14003.

33. Tashkin DP, Celli B, Senn S, Burkhart D, Kesten S, Menjoge S. A 4-year trial of tiotropium in chronic obstructive pulmonary disease. N Engl J Med. 2008;359(15):1543-1554.

34. Celli B, Decramer M, Kesten S, Liu D, Mehra S, Tashkin DP. Mortality in the 4-year trial of tiotropium (UPLIFT) in patients with chronic obstructive pulmonary disease. Am J Respir Crit Care Med. 2009;180(10):948-955.

35. Verkindre C, Bart F, Aguilaniu B, et al. The effect of tiotropium on hyperinflation and exercise capacity in chronic obstructive pulmonary disease. Respiration. 2006;73(4):420-427.

36. Zhou Y, Zhong NS, Li X, et al. Tiotropium in early-stage chronic obstructive pulmonary disease. $N$ Engl J Med. 2017;377(10):923-935.

37. Vincken W, van Noord JA, Greefhorst AP, Bantje TA, Kesten S, Korducki L. Improved health outcomes in patients with COPD during 1 yr's treatment with tiotropium. Eur Respir J. 2002;19(2):209-216.

38. van Noord JA, Bantje TA, Eland ME, Korducki L, Cornelissen PJ. A randomised controlled comparison of tiotropium nd ipratropium in the treatment of chronic obstructive pulmonary disease. The Dutch Tiotropium Study Group. Thorax. 2000;55(4):289-294.

39. Bateman E, Singh D, Smith D, et al. Efficacy and safety of tiotropium Respimat SMI in COPD in two 1-year randomized studies. Int J Chron Obstruct Pulmon Dis. 2010;5:197-208.

40. Bateman ED, Tashkin D, Siafakas N, et al. A one-year trial of tiotropium Respimat plus usual therapy in COPD patients. Respir Med. 2010;104(10):1460-1472.

41. Voshaar T, Lapidus R, Maleki-Yazdi R, et al. A randomized study of tiotropium Respimat Soft Mist inhaler vs. ipratropium pMDI in COPD. Respir Med. 2008;102(1):32-41.

42. Wise RA, Anzueto A, Calverley P, et al. The Tiotropium Safety and Performance in Respimat Trial (TIOSPIR), a large scale, randomized, controlled, parallel-group trial-design and rationale. Respir Res. 2013;14:40.

43. Wise RA, Anzueto A, Cotton D, Dahl R, Devins T, Disse B. Tiotropium Respimat inhaler and the risk of death in COPD. N Engl J Med. 2013;369(16):1491-1501

44. Anzueto A, Wise R, Calverley P, et al. The Tiotropium Safety and Performance in Respimat ${ }^{\circledR}$ (TIOSPIR $^{\circledR}$ ) Trial: spirometry outcomes. Respir Res. 2015;16:107.

45. Trivedi R, Richard N, Mehta R, Church A. Umeclidinium in patients with COPD: a randomised, placebo-controlled study. Eur Respir J. 2014;43(1):72-81.

46. Feldman G, Maltais F, Khindri S, et al. A randomized, blinded study to evaluate the efficacy and safety of umeclidinium $62.5 \mu \mathrm{g}$ compared with tiotropium $18 \mu \mathrm{g}$ in patients with COPD. Int J Chron Obstruct Pulmon Dis. 2016;11:719-730.

47. Rheault T, Khindri S, Vahdati-Bolouri M, Church A, Fahy WA. A randomised, open-label study of umeclidinium versus glycopyrronium in patients with COPD. ERJ Open Res. 2016;2(2).

48. D'Urzo AD, Rennard SI, Kerwin EM, Mergel V, Leselbaum AR, Caracta CF; AUGMENT COPD study investigators. Efficacy and safety of fixed-dose combinations of aclidinium bromide/formoterol fumarate: the 24-week, randomized, placebo-controlled AUGMENT COPD study. Respir Res. 2014;15:123.

49. Singh D, Jones PW, Bateman ED, et al. Efficacy and safety of aclidinium bromide/formoterol fumarate fixed-dose combinations compared with individual components and placebo in patients with COPD (ACLIFORM-COPD): a multicentre, randomised study. BMC Pulm Med. 2014;14:178 
50. Vogelmeier C, Paggiaro PL, Dorca J, et al. Efficacy and safety of aclidinium/formoterol versus salmeterol/fluticasone: a Phase 3 COPD study. Eur Respir J. 2016;48(4):1030-1039.

51. Bateman ED, Ferguson GT, Barnes N, et al. Dual bronchodilation with QVA149 versus single bronchodilator therapy: the SHINE study. Eur Respir J. 2013;42(6):1484-1494.

52. Dahl R, Chapman KR, Rudolf M, et al. Safety and efficacy of dual bronchodilation with QVA149 in COPD patients: the ENLIGHTEN study. Respir Med. 2013;107(10):1558-1567.

53. Vogelmeier CF, Bateman ED, Pallante J, et al. Efficacy and safety of once-daily QVA149 compared with twice-daily salmeterol-fluticasone in patients with chronic obstructive pulmonary disease (ILLUMINATE): a randomised, double-blind, parallel group study. Lancet Respir Med. 2013;1(1):51-60.

54. Wedzicha JA, Banerji D, Chapman KR, Vestbo J, Roche N, Ayers RT. Indacaterol-glycopyrronium versus salmeterol-fluticasone for COPD. N Engl J Med. 2016;374(23):2222-2234.

55. Zhong N, Wang C, Zhou X, Zhang N, Humphries M, Wang L. LANTERN: a randomized study of QVA149 versus salmeterol/fluticasone combination in patients with COPD. Int J Chron Obstruct Pulmon Dis. 2015;10:1015-1026.

56. Wedzicha JA, Decramer M, Ficker JH, et al. Analysis of chronic obstructive pulmonary disease exacerbations with the dual bronchodilator QVA149 compared with glycopyrronium and tiotropium (SPARK): a randomised, double-blind, parallel-group study. Lancet Respir Med. 2013;1(3):199-209.

57. Singh D, Ferguson GT, Bolitschek J, et al. Tiotropium + olodaterol shows clinically meaningful improvements in quality of life. Respir Med. 2015;109(10):1312-1319.

58. Buhl R, Maltais F, Abrahams R, et al. Tiotropium and olodaterol fixeddose combination versus mono-components in COPD (GOLD 2-4). Eur Respir J. 2015;45(4):969-979.

59. Buhl R, Magder S, Bothner U, et al. Long-term general and cardiovascular safety of tiotropium/olodaterol in patients with moderate to very severe chronic obstructive pulmonary disease. Respir Med. 2017;122:58-66.

60. Kerwin EM, Kalberg CJ, Galkin DV, et al. Umeclidinium/vilanterol as step-up therapy from tiotropium in patients with moderate COPD: a randomized, parallel-group, 12-week study. Int J Chron Obstruct Pulmon Dis. 2017;12:745-755.

61. Siler TM, Donald AC, O'Dell D, Church A, Fahy WA. A randomized, parallel-group study to evaluate the efficacy of umeclidinium/vilanterol $62.5 / 25 \mu \mathrm{g}$ on health-related quality of life in patients with COPD. Int J Chron Obstruct Pulmon Dis. 2016;11:971-979.

62. Zheng J, Zhong N, Newlands A, Church A, Goh AH. Efficacy and safety of once-daily inhaled umeclidinium/vilanterol in Asian patients with COPD: results from a randomized, placebo-controlled study. Int J Chron Obstruct Pulmon Dis. 2015;10:1753-1767.

63. Donohue JF, Maleki-Yazdi MR, Kilbride S, Mehta R, Kalberg C, Church A. Efficacy and safety of once-daily umeclidinium/vilanterol 62.5/25 mcg in COPD. Respir Med. 2013;107(10):1538-1546.

64. Decramer M, Anzueto A, Kerwin E, et al. Efficacy and safety of umeclidinium plus vilanterol versus tiotropium, vilanterol, or umeclidinium monotherapies over 24 weeks in patients with chronic obstructive pulmonary disease: results from two multicentre, blinded, randomised controlled trials. Lancet Respir Med. 2014;2(6):472-486.

65. Maleki-Yazdi MR, Kaelin T, Richard N, Zvarich M, Church A. Efficacy and safety of umeclidinium/vilanterol $62.5 / 25 \mathrm{mcg}$ and tiotropium $18 \mathrm{mcg}$ in chronic obstructive pulmonary disease: results of a 24-week, randomized, controlled trial. Respir Med. 2014;108(12):1752-1760.

66. Donohue JF, Worsley S, Zhu CQ, Hardaker L, Church A. Improvements in lung function with umeclidinium/vilanterol versus fluticasone propionate/salmeterol in patients with moderate-to-severe COPD and infrequent exacerbations. Respir Med. 2015;109(7):870-881.

67. Singh D, Worsley S, Zhu CQ, Hardaker L, Church A. Umeclidinium/ vilanterol versus fluticasone propionate/salmeterol in COPD: a randomised trial. BMC Pulm Med. 2015;15:91.
68. Tashkin DP, Rennard SI, Martin P, et al. Efficacy and safety of budesonide and formoterol in one pressurized metered-dose inhaler in patients with moderate to very severe chronic obstructive pulmonary disease: results of a 6-month randomized clinical trial. Drugs. 2008;68(14):1975-2000.

69. Rennard SI, Tashkin DP, Mcelhattan J, et al. Efficacy and tolerability of budesonide/formoterol in one hydrofluoroalkane pressurized metereddose inhaler in patients with chronic obstructive pulmonary disease: results from a 1-year randomized controlled clinical trial. Drugs. 2009;69(5):549-565.

70. Bhatt SP, Dransfield MT, Cockcroft JR, et al. A randomized trial of once-daily fluticasone furoate/vilanterol or vilanterol versus placebo to determine effects on arterial stiffness in COPD. Int $J$ Chron Obstruct Pulmon Dis. 2017;12:351-365.

71. Kerwin EM, Scott-Wilson C, Sanford L, et al. A randomised trial of fluticasone furoate/vilanterol $(50 / 25 \mu \mathrm{g} ; 100 / 25 \mu \mathrm{g})$ on lung function in COPD. Respir Med. 2013;107(4):560-569.

72. Martinez FJ, Boscia J, Feldman G, et al. Fluticasone furoate/vilanterol $(100 / 25 ; 200 / 25 \mu \mathrm{g})$ improves lung function in COPD: a randomised trial. Respir Med. 2013;107(4):550-559.

73. Covelli H, Pek B, Schenkenberger I, Scott-Wilson C, Emmett A, Crim C. Efficacy and safety of fluticasone furoate/vilanterol or tiotropium in subjects with COPD at cardiovascular risk. Int J Chron Obstruct Pulmon Dis. 2016;11:1-12.

74. Pepin JL, Cockcroft JR, Midwinter D, Sharma S, Rubin DB, Andreas S. Long-acting bronchodilators and arterial stiffness in patients with COPD: a comparison of fluticasone furoate/vilanterol with tiotropium. Chest. 2014;146(6):1521-1530.

75. Dransfield MT, Feldman G, Korenblat P, et al. Efficacy and safety of once-daily fluticasone furoate/vilanterol $(100 / 25 \mathrm{mcg})$ versus twicedaily fluticasone propionate/salmeterol $(250 / 50 \mathrm{mcg})$ in COPD patients. Respir Med. 2014;108(8):1171-1179.

76. Agustí A, de Teresa L, de Backer W, et al. A comparison of the efficacy and safety of once-daily fluticasone furoate/vilanterol with twice-daily fluticasone propionate/salmeterol in moderate to very severe COPD. Eur Respir J. 2014;43(3):763-772.

77. Asai K, Kobayashi A, Makihara Y, Johnson M. Anti-inflammatory effects of salmeterol/fluticasone propionate $50 / 250 \mathrm{mcg}$ combination therapy in Japanese patients with chronic obstructive pulmonary disease. Int J Chron Obstruct Pulmon Dis. 2015;10:803-811.

78. Hanania NA, Darken P, Horstman D, et al. The efficacy and safety of fluticasone propionate $(250 \mathrm{microg}) / \mathrm{salmeterol}(50 \mathrm{microg}) \mathrm{com}-$ bined in the Diskus inhaler for the treatment of COPD. Chest. 2003; 124(3):834-843.

79. Mahler DA, Wire P, Horstman D, et al. Effectiveness of fluticasone propionate and salmeterol combination delivered via the Diskus device in the treatment of chronic obstructive pulmonary disease. Am J Respir Crit Care Med. 2002;166(8):1084-1091.

80. Calverley PM, Anderson JA, Celli B, et al. Salmeterol and fluticasone propionate and survival in chronic obstructive pulmonary disease. N Engl J Med. 2007;356(8):775-789.

81. Jones PW, Anderson JA, Calverley PM, et al. Health status in the TORCH study of COPD: treatment efficacy and other determinants of change. Respir Res. 2011;12:71.

82. Jenkins CR, Jones PW, Calverley PM, et al. Efficacy of salmeterol/ fluticasone propionate by GOLD stage of chronic obstructive pulmonary disease: analysis from the randomised, placebo-controlled TORCH study. Respir Res. 2009;10:59.

83. Zheng JP, Yang L, Wu YM, et al. The efficacy and safety of combination salmeterol (50 microg)/fluticasone propionate (500 microg) inhalation twice daily via accuhaler in Chinese patients with COPD. Chest. 2007;132(6):1756-1763.

84. Cazzola M, Andò F, Santus P, et al. A pilot study to assess the effects of combining fluticasone propionate/salmeterol and tiotropium on the airflow obstruction of patients with severe-to-very severe COPD. Pulm Pharmacol Ther. 2007;20(5):556-561. 
85. Perng DW, Tao CW, Su KC, Tsai CC, Liu LY, Lee YC. Anti-inflammatory effects of salmeterol/fluticasone, tiotropium/fluticasone or tiotropium in COPD. Eur Respir J. 2009;33(4):778-784.

86. Dahl R, Greefhorst LA, Nowak D, et al. Inhaled formoterol dry powder versus ipratropium bromide in chronic obstructive pulmonary disease. Am J Respir Crit Care Med. 2001;164(5):778-784.

87. Taylor J, Kotch A, Rice K, et al. Ipratropium bromide hydrofluoroalkane inhalation aerosol is safe and effective in patients with COPD. Chest. 2001;120(4):1253-1261.

88. Sion KY, Huisman EL, Punekar YS, Naya I, Ismaila AS. A network meta-analysis of long-acting muscarinic antagonist (LAMA) and longacting $\beta 2$-agonist (LABA) combinations in COPD. Pulmonary Therapy. 2017;3(2):297-316.

89. Cope S, Donohue JF, Jansen JP, et al. Comparative efficacy of longacting bronchodilators for COPD-a network meta-analysis. Respiratory research. 2013;14(1):100.

90. Karabis A, Lindner L, Mocarski M, Huisman E, Greening A. Comparative efficacy of aclidinium versus glycopyrronium and tiotropium, as maintenance treatment of moderate to severe COPD patients: a systematic review and network meta-analysis. Int J Chron Obstruct Pulmon Dis. 2013;8:405.
91. Ismaila AS, Huisman EL, Punekar YS, Karabis A. Comparative efficacy of long-acting muscarinic antagonist monotherapies in COPD: a systematic review and network meta-analysis. Int J Chron Obstruct Pulmon Dis. 2015;10:2495.

92. Schlueter M, Gonzalez-Rojas N, Baldwin M, Groenke L, Voss F, Reason T. Comparative efficacy of fixed-dose combinations of longacting muscarinic antagonists and long-acting $\beta 2$-agonists: a systematic review and network meta-analysis. Ther Adv Respir Dis. 2016;10(2): 89-104.

93. Huisman EL, Cockle SM, Ismaila AS, Karabis A, Punekar YS. Comparative efficacy of combination bronchodilator therapies in COPD: a network meta-analysis. Int J Chron Obstruct Pulmon Dis. 2015;10:1863

International Journal of COPD

\section{Publish your work in this journal}

The International Journal of COPD is an international, peer-reviewed journal of therapeutics and pharmacology focusing on concise rapid reporting of clinical studies and reviews in COPD. Special focus is given to the pathophysiological processes underlying the disease, intervention programs, patient focused education, and self management protocols.

\section{Dovepress}

This journal is indexed on PubMed Central, MedLine and CAS. The manuscript management system is completely online and includes a very quick and fair peer-review system, which is all easy to use. Visit http://www.dovepress.com/testimonials.php to read real quotes from published authors.

Submit your manuscript here: http://www.dovepress.com/international-journal-of-chronic-obstructive-pulmonary-disease-journal 